\title{
Anabases
}

ANABASES Traditions et réceptions de l'Antiquité

$2 \mid 2005$

Varia

\section{Marx, Weber et l'esclavage}

Wilfried Nippel

\section{CpenEdition}

Journals

Édition électronique

URL : http://journals.openedition.org/anabases/1531

DOI : 10.4000/anabases. 1531

ISSN : 2256-9421

\section{Éditeur}

E.R.A.S.M.E.

\section{Édition imprimée}

Date de publication : 1 octobre 2005

Pagination : 11-52

ISSN : 1774-4296

\section{Référence électronique}

Wilfried Nippel, «Marx, Weber et l'esclavage », Anabases [En ligne], 2 | 2005, mis en ligne le 01 juillet 2011, consulté le 14 novembre 2019. URL : http://journals.openedition.org/anabases/1531

(c) Anabases 
Anabases 2 (2005), p. 11-52

\title{
Marx, Weber et l'esclavage*
}

WiLFRIED NiPPEL

\begin{abstract}
À SES DÉBUTS, la discussion moderne au sujet de l'esclavage antique consista essentiellement en une réaction aux débats, devenus de plus en plus intenses au XVIII siècle, sur la légitimité et l'utilité de l'esclavage dans les colonies d'Outre-Mer et dans les ÉtatsUnis d'Amérique, ainsi que sur celles du trafic intercontinental d'êtres humains qui y était lié ${ }^{1}$.
\end{abstract}

La version originale de cette étude a été publiée sous le titre "Marx, Weber und die Sklaverei", in Elisabeth HERRMANN-OTTO (éd.), Unfreie Arbeits- und Lebensverhältnisse von der Antike bis in die Gegenwart (Sklaverei. Knechtschaft. Zwangsarbeit. Untersuchungen zur Sozial-, Rechts- und Kulturgeschichte, vol. 1), Hildesheim, Olms, 2005, p. 317-356. Traduction d'Anne-Laure Vignaux, revue par Hinnerk Bruhns.

Le système de citation de l'auteur et la volumineuse bibliographie in fine, très utile, ont été gardés pour la version française. Les citations reprises en traduction dans une version éditée sont signalées dans les notes de bas de page entre crochets par le début du titre français précédé de la mention "trad. in "; la référence complète à la traduction peut être trouvée dans la bibliographie, sous le titre original allemand. Pour Marx et Engels, auxquels l'auteur ne fait référence qu'à travers les éditions allemandes des œuvres complètes (abrégées MEW ou MEGA), nous avons en outre mentionné, dans le souci d'orienter le lecteur, le titre de l'œuvre en français (sauf si ce titre était déjà nommé dans le passage de texte correspondant) ; ces titres n'ont pas été intégrés dans la bibliographie. Si aucun auteur n'est mentionné, il s'agit de Marx. Pour Weber, les renvois aux différentes éditions allemandes (GARS, GASS, GASWG, GAWL, GPS, MWG, Wg, WuG) ont été complétés par l'indication des traductions françaises quand elles existent. Dans certains cas, les citations prises dans des traductions françaises de Marx, Engels, Smith ou Weber ont été modifiées ou corrigées dans un souci de clarté. Ces interventions de $\mathrm{H}$. Bruhns sont signalées par la mention " trad. mod. ». Pour les auteurs français cités par l'auteur dans des traductions allemandes, la référence à l'édition française est ajoutée entre parenthèses. DAVIS (1966) constitue à cet égard un texte fondateur. 


\section{Abolitionnisme - Morale et économie politique}

L'Antiquité entre en jeu à des titres divers dans ces débats. Les défenseurs de l'esclavage, notamment dans le Sud des États-Unis, reprirent - outre la référence à l'esclavage en tant qu'institution du droit romain et du droit des gens - la catégorie aristotélicienne de l'« esclavage naturel 2 ». Bien que déjà réexhumée au XVIe siècle dans le cadre de la discussion espagnole sur le colonialisme, cette théorie n’avait pas réussi à s'imposer face à la critique de théologiens et de juristes comme Vitoria et l'école de Salamanque, ainsi que Las Casas ${ }^{3}$. Elle trouva de nouveaux points d'appui dans l'anthropologie physique, développée à partir du XVIII siècle qui faisait de la couleur de la peau et de la race le critère distinctif ${ }^{4}$, ce qui permettait, pensait-on, d'assimiler les Noirs africains à ces " esclaves par nature " qui avaient besoin, dans leur propre intérêt, d'être soumis à un maître ${ }^{5}$. L'identification des Africains avec les Chamites, dont l'esclavage perpétuel trouvait une légitimation biblique dans la malédiction lancée par Noé contre les descendants de son fils $\mathrm{Cham}^{6}$, ne fit que renforcer cette théorie. D’une manière générale, on put invoquer le fait que l'esclavage était en accord avec la tradition biblique et correspondait aussi à la conception chrétienne patriarcale de la famille. Dans l'ensemble, le recours à de telles stratégies pour légitimer l'esclavage se produisit particulièrement lorsque les défenseurs de cette institution étaient sur la défensive face à l'abolitionnisme et qu'ils s'obstinaient d'autant plus dans la voie de l'esclavagisme qu'à travers son maintien, c'était un ordre social légitime qu'ils défendaient ${ }^{7}$.

2 Aristote développa cette théorie dans des textes polémiques par rapport à d'autres positions ; malheureusement, ce contexte contemporain ne peut être que partiellement restitué ; voir CAMBIANO (1987).

3 Cf. NipPel (1990a), 30 sv. ; IDEM. (1993) ; IDEM (2000c), avec des références détaillées.

4 GREENE (1954) : JORDAN (1968), 216 sv. - En Allemagne, la réception de la conception selon laquelle la libération des esclaves était, en raison des caractéristiques raciales des Noirs, insensée et dangereuse, est surtout due à Christoph Meiners, philosophe de Göttingen, connu comme polygraphe travaillant toujours de seconde main ; MEINERS (1790/1997).

5 Wish (1949) ; BackHaus (1974) 98 sv. ; Hartfield (1983) ; Harrington (1989).

6 MCKeE Evans (1980) ; Haynes (2002). - Sur les origines de cette tradition, voir GOLDENBERG (2003).

7 Backhaus (1975) 547 ; FoX-Genovese - Genovese (1987) ; Osterhammel (2000) 46 sv. ; KolChin (2003) 190 sv. - Cairnes a décrit cette attitude sudiste dans CAIRNES (1863/2003) 167 : Lesclavage " is now propounded as a great discovery in ethical and political science, made for the first time by the enlightened leaders of the Southern Confederation, and recommended by that philantropic body to all civilized nations for their adoption "; sur Cairnes, voir infra. - Dans le Capital, Marx cite l'exemple d'une manifestation qui se déroula à New York en 1859 avec pour mot d'ordre : "Justice pour le Sud »; MEW 25, 399. 
Les plaidoyers en faveur de l'abolition de l'esclavage furent d'abord l'œuvre de quakers et de mennonites puis, plus tard, de groupes évangéliques ${ }^{8}$, comme les méthodistes. Ils se nourrissaient du concept chrétien de l'amour du prochain, qui impliquait aussi une amélioration de la morale des couches inférieures de la société par le biais d'un travail censé remplacer l'oisiveté et la consommation d'alcool ${ }^{9}$. En Grande-Bretagne, cet abolitionnisme se développa pour devenir l'un des «mouvements de masse les plus puissants de l'histoire moderne 10 »; grâce à des pétitions au Parlement, à des boycotts et à une série d'autres activités, il obtiendra de fait, en 1807, l'abolition de la traite dans l'Empire britannique et, en 1833, la suppression de l'esclavage dans les colonies anglaises ${ }^{11}$. À chacune de ses étapes, l'évolution de la question en Angleterre eut de fortes répercussions sur la situation aux États-Unis. Les opposants de l'esclavage se virent ainsi renforcés, tandis que ses partisans flairèrent là de perfides machinations d'Albion contre la souveraineté des États-Unis ${ }^{12}$.

Toutefois, l'argumentation chrétienne contre le maintien de l'esclavage se heurtait toujours à l'objection selon laquelle ni le christianisme primitif ${ }^{13}$, ni l'Église du Moyen Âge ${ }^{14}$ ou du début des Temps modernes n'avaient fondamentalement remis en cause l'institution comme telle. C'est encore à cette objection que dut répondre Henri Wallon, qui étaya son engagement politique en faveur de la suppression de l'esclavage

8* Le terme anglais evangelical prend, à partir du XVIII siècle, un sens particulier et désigne des mouvements dont le point de départ est l'expérience de la conversion intérieure. Cf. Encyclopédie du Protestantisme, Paris, 1995, p. 551 (note ajoutée par HB).

9 Les idées de William Wilberforce illustrent parfaitement le lien établi entre ces deux aspects. Wilberforce fut, pendant des décennies, l'orateur vedette de l'abolitionnisme au parlement britannique.

10 BERDING (1974) 271.

11 DAVIS (1975) ; DRESCHER (1994). - Le Danemark avait été le premier pays à abolir l'esclavage en 1803 ; le congrès de Vienne vota en 1815 une déclaration contre la traite des esclaves ; BERDING (1974).

12 MASON (2002)

13 Pour les débuts du christianisme, l'épître à Philémon de Paul, avec ses ambivalences, est représentative ; cf. (parmi une abondante littérature) BARCLAY 1991. - Chez les Pères de l'Église plus tardifs, l'attaque la plus violente contre l'institution de l'esclavage apparaît chez Grégoire de Nysse, qui décrit comme un péché l'esclavage de l'homme, créé par Dieu à son image ; il ne va toutefois pas jusqu'à prêcher l'abolition ni à encourager ses auditeurs chrétiens à affranchir tous leurs esclaves ; GARNSEY (1996) 80 sv.

14 Les canonistes du Moyen Âge développèrent, il est vrai, la doctrine selon laquelle les chrétiens ne devraient pas réduire d'autres chrétiens à l'esclavage, si bien que, même en temps de guerre, ils devraient renoncer à épuiser entièrement les possibilités du droit coutumier des gens ; RuSSELL (1975) 120, 162 et 279 ; pour la persistance de cette tradition, voir par ex. Grotius, De jure belli ac pacis (1625), Livre III, Chap. 7, $\$ 9$ = GrOTIUs (1950), 484. 
dans les colonies françaises 15 par une imposante collecte de matériel sur l'esclavage antique publiée en $18477^{16}$ et fut, en 1848, membre de la Commission qui rédigea la loi sur l'abolition de l'esclavage dans ces territoires. Dans son travail sur l'Antiquité, Wallon développa l'argument selon lequel, si le christianisme n'avait pas fondamentalement délégitimé l'institution, il avait du moins encouragé un traitement humain des esclaves. On peut ainsi établir une filiation directe entre Wallon et Joseph Vogt, Sklaverei und Humanität 17.

Outre cette argumentation philanthropico-chrétienne, on constate, au XVIII' siècle, l'émergence, en particulier dans la philosophie sociale et l'économie politique écossaises, d'un second schéma d'argumentation : selon celui-ci, l'esclavage n'était pas rentable d'un point de vue économique et son efficacité était inférieure à celle du travail libre salarié, si bien qu'il représentait un obstacle sur la voie menant à la société commerciale.

D'après John Millar (The Origin of the Distinction of Ranks, 1779), un esclave qui travaille sous la contrainte et reçoit de toute façon sa nourriture ne peut fournir un travail efficace et est inapte à des activités plus ambitieuses ${ }^{18}$. Vu le coût d'acquisition et d'entretien des esclaves, il est clair que "le travail d'un esclave, qui ne reçoit rien d'autre que son simple entretien, est en réalité plus cher que le travail d'un homme libre qui perçoit un salaire régulier en fonction de ses prestations ${ }^{19}$ ".

Dans son Enquête sur la nature et les causes de la richesse des nations (1776), Adam Smith défend l'idée suivante : "[...] l'expérience de toutes les époques et de toutes les nations montre donc, me semble-t-il, que l'ouvrage fait par des hommes libres revient en définitive moins cher que celui exécuté par des esclaves ${ }^{20}$. "Des salaires relative-

15 Le «Code Noir » de Louis XIV, en 1685, autorisait l'esclavage dans le domaine colonial français en arguant du fait qu'il fallait élever les esclaves dans la foi catholique. Pendant la Révolution française, la question de l'esclavage fut, en dépit de la critique antérieure (certes modérée) de l'esclavage par les Lumières (cf. HunTING 1978) et malgré la déclaration des Droits de l'homme de septembre 1789, traitée de manière avant tout dilatoire ; l'initiative des Girondins (Brissot ; Abbé de Grégoire) en vue d'abolir l'esclavage fut contrecarrée par les Jacobins, pour qui la défense de la révolution et de la patrie était prioritaire. L'abolition soudaine de l'esclavage par la Convention nationale en février 1794 était une réaction à la révolte des esclaves de Saint-Domingue (Haïti). L'esclavage fut réintroduit dans les colonies françaises en 1802, avant d'être aboli en 1848 ; cf. RESNICK (1972) ; DumOnt (1988) ; IDEM (1992) ; BENOT (1988) ; BLACKBURN (1988) chap. V, VI et XII ; GEGGUS (1989). Wallon (1847/1879) ; cf. Biezunska Malowist (1968) 164 sv. ; Vogt (1972) 98 sv. ; FinLEY (1981a) 13 sv. et 37 sv. ; RUBINSOHN (1993) 36 sv. VOGT (1953) ; IDEM (1972).

18 Chap. VI, sect. II ; Millar (1806) 250.

19 Ibid.; Millar (1806) 255.

20 A. SMITH (1776/1981) Livre I, Chap. 8 [trad. in Enquête sur la nature 294] ; cf. aussi Livre III, Chap. 2. - Le premier passage est également cité par Engels ; MEW 2, 310 sv. [ENGELS, La Situation de la classe laborieuse en Angleterre]. 
ment élevés pour les ouvriers libres favorisaient en effet la productivité et l'accroissement démographique ${ }^{21}$. Les esclaves, en revanche, ne s'embarrassaient pas d'innovations techniques ou d'amélioration de l'organisation du travail, car leur maître aurait réprimé ces initiatives comme des signes de paresse. "Dans les manufactures assurées par les esclaves, on a donc dû employer généralement plus de travailleurs à exécuter la même quantité d'ouvrage que dans celles assurées par des hommes libres. L'ouvrage des premiers a dû, pour cette raison, être plus cher que celui des seconds ${ }^{22}$. " Les mesures économiquement pertinentes en faveur d'une protection des esclaves par l'État se heurtant en outre aux droits de propriété, elles n'étaient possibles que dans les régimes autoritaires. À l'époque, ce fait se vérifiait dans le contraste entre, d'une part, la France et ses colonies, où l'arbitraire de l'État et la protection des esclaves allaient de pair, et, d'autre part, la Grande-Bretagne, caractérisée par un régime libéral et l'absence de mesures de protection dans les colonies ; dans le passé, ce dilemme était confirmé par la différence entre le régime des esclaves romains sous l'Empire et sous la République. "Que la condition d'un esclave soit meilleure sous un gouvernement arbitraire que sous un gouvernement libre est, je crois, confirmé par l'histoire de tous les temps et de toutes les nations 23 ."

David Hume avait déjà, dans son célèbre essai de 1752 sur la démographie dans l'ancien monde (De la populosité des nations antiques ${ }^{24}$ ), développé la thèse selon laquelle l'esclavage correspondait au caractère guerrier des anciennes sociétés et avait été nuisible à la croissance démographique et au progrès économique en raison du coût trop élevé de l'élevage des esclaves. Hume contestait également, dans ce cadre, le chiffre, mentionné chez Athénée et fondé sur le recensement de Démétrios de Phalère à la fin du IVe siècle av. J.-C., de 400000 esclaves à Athènes (pour 21000 citoyens et 10000 métèques 25 ) ; il devait, selon lui, s'agir de 40 000. Hume tire l'un de ses arguments d'une comparaison avec les colonies britanniques d'Amérique du Nord : un excédent, si petit soit-il, d'esclaves par rapport à la population libre impose un contrôle serré, quasi militaire, de ceux-ci.

Plus tard, pour Thomas Jefferson, la répression nécessaire des masses d'esclaves allait être, comme on le sait, un motif pour ne faire suivre son refus théorique de l'esclavage d'aucune démarche concrète en vue de son abolition ${ }^{26}$. On « tenait un loup

Ibid., Livre I, Chap. 8.

Ibid., Livre IV, Chap. 8 [trad. in Enquête sur la nature 781, trad. mod.]

Ibid., Livre IV, Chap. 7 [trad. in Enquête sur la nature 673] - Cf. aussi MARX et ENGELS (Deutsche Ideologie, 1845) : "Un yankee se rend en Angleterre ; le juge de paix l'empêche de fouetter son esclave et il s'écrie, indigné : "Do you call this a land of liberty, where a man can't larrup his nigger ?” "; MEW 3, 191 [trad. in L'Idéologie allemande 203]. Hume (1882). Athénée 272c.

Au sujet de la position ambiguë de Jefferson, voir par ex. Diggins (1976) ; Wasser (1996) ; FinKELMAN (1996) ; TEMPERLEY (1997) ; SPAHN (2002). 
par les oreilles "; une émancipation des esclaves noirs reviendrait à libérer une armée de pauvres, qui menacerait la propriété privée et déclencherait une guerre civile 27 . La conviction largement répandue selon laquelle les esclaves noirs ne pourraient pas être intégrés à la société après leur libération fit que l'on s'efforça d'organiser leur retour en Afrique, plus précisément dans les établissements fondés à partir de 1822 par l'American Colonization Society (et par d'autres organisations), dans le futur Liberia 28.

À l'époque de la fondation des États-Unis, on avait craint qu'une abolition de l'esclavage ne mette en danger l'indépendance ${ }^{29}$ et la cohésion de l'Union. On avait donc, dans la constitution fédérale de 1787/88, sciemment exclu la question de l'esclavage 30 , même si on n'ignorait pas qu'étant donné la mise en exergue des droits de l'homme dans la Déclaration d'indépendance et dans les Déclarations des droits fondamentaux des États individuels, comme le Bill of Rights (1776) de Virginie, le thème serait remis à l'ordre du jour et pourrait difficilement être éludé une nouvelle fois 31 . Pour une durée de vingt ans, toute loi fédérale sur la traite des esclaves fut exclue. Au terme de ce délai, en 1808, l'importation d'esclaves fut interdite.

Les affirmations de Hume, Smith et d'autres sur la productivité a priori plus faible du travail des esclaves comparée à celle du travail libre se fondaient non pas sur des données fiables, mais bien sur certaines impressions, qu'ils amplifiaient jusqu’à en faire des propositions quasi-axiomatiques. Leur argumentation sera néanmoins reprise dans

27 Lettre de Jefferson à John Holmes, 4. 8. 1820 : la solution au problème de l'esclavage devait résider, à long terme, dans " a general emancipation and expatriation "; " but as it is, we have the wolf by the ears, and we can neither hold him, nor safely let him go. Justice is in one scale, and self-preservation in the other" ; JEFFERSON (1984) 1434. - Jefferson adapte ici une métaphore de Plutarque, Moralia 802D ; SHAW (1998) 4. - La crainte des conséquences d'un affranchissement des esclaves s'était également accrue à la suite des combats sanglants qui avaient eu lieu à Haïti après la prise d'indépendance en 1804. Des observateurs contemporains se montrèrent en revanche surpris du déroulement généralement peu problématique de l'affranchissement des esclaves dans les colonies anglaises; cf. MOHL (1844).

28 DAVIS (2003) 62 sv. - Il y avait eu, au préalable, la fondation, en 1787, de la colonie de Freetown (colonie de la Couronne à partir de 1806), au Sierra Leone, comme lieu d'établissement pour les esclaves affranchis.

29 L'exportation de tabac, en particulier vers la France, était la principale source de financement de la guerre d'indépendance ; le tabac venait principalement des plantations de Virginie, qui étaient exploitées grâce à des esclaves africains.

30 Il est vrai que les États sudistes avaient imposé le fait que, pour les sièges de la chambre des représentants revenant aux États individuels, on compte non seulement les citoyens libres, mais aussi "trois cinquièmes de toutes les autres personnes"; le terme d' " esclaves " fut soigneusement évité.

31 La formule de compromis, selon laquelle les droits fondamentaux ne pouvaient valoir que pour les membres de la société à laquelle les esclaves n'appartenaient évidemment pas, ne put apaiser cette tension ; cf. ADAMS (1971) ; E.S. MORGAN (1972/73) ; STOURZH (1989) ; NiEMAN (1991); K. MORGAN (2001). 
le répertoire standard de l'abolitionnisme (et de l'économie politique). Le XIX ${ }^{e}$ siècle produisit une abondante littérature dans laquelle on réclamait non seulement l'abolition de l'esclavage pour des motifs éthiques et chrétiens, ainsi qu'au nom des droits de l'homme, mais où l'on mettait aussi en avant les inconvénients économiques de ce système par rapport au travail exécuté par des hommes libres ${ }^{32}$. La force de conviction de l'abolitionnisme s'explique précisément par le fait qu'il ne se fondait pas seulement sur des arguments philanthropiques, mais qu'il se réclamait aussi de la rationalité économique.

Dans le Sud des États-Unis, l'hypothèse de l'inefficacité économique de l'esclavage fut toutefois reprise par ses défenseurs, qui se targuaient d'adopter, vis-à-vis de leurs esclaves, une attitude paternaliste de prise en charge à vie, alors que les employeurs du Nord engageaient et licenciaient de la main d'œuvre selon leurs besoins et cherchaient sans cesse à diminuer les salaires 33 .

On s'aperçoit donc que les questions de l'efficacité économique, de l'impact démographique et de la stabilité sociale se sont, dès le début, entremêlées à plusieurs niveaux dans le débat moderne sur l'esclavage. Ce phénomène s'est prolongé au XXe siècle et s'applique également à la discussion menée aux États-Unis depuis la fin des années 1950. Quelle que soit la valeur intrinsèque de l'aperçu général 34 offert par les cliométriciens Fogel et Engerman ${ }^{35}$, ces derniers ont en tout cas réfuté la thèse d'une inefficacité économique générale de l'esclavage pour les États esclavagistes des ÉtatsUnis ${ }^{36}$. Nous reviendrons sur ce point.

Les exemples de Marx et Engels et, à d'autres égards, de Max Weber permettent de montrer que le caractère d'arriération économique attribué au cours du XIXe siècle à l'esclavage moderne a longtemps influencé le débat sur l'esclavage antique.

32 Huston (2000) ; DresCher (2002). - En 1826, Alexander von Humboldt avait par exemple plaidé avec des arguments économiques pour l'abolition de l'esclavage dans les Caraïbes ; RAPHAEL (1995).

33 Par exemple FitzHUGH (1857/1988) 201 : « Our Southern slavery has become a benign and protective institution, and our negroes are confessedly better off than any free laboring population in the world. »Ibid., 220 : "We [les Sudistes] love our slaves, and we are ready to defend, assist and protect them ; you [Yankees] hate and fear your white servants, and never fail, as a moral duty, to screw down their wages to the lowest [...]. "Ibid., 19 : "Free laborers have not a thousandst part of the rights and liberties of negro slaves. Indeed, they have not a single liberty, unless it be the right or liberty to die. " - Voir en général WoOdman (1963) ; BACKHAUs (1974) 89 sv. ; PATTERSON (1998) 175 sv. L'énorme impact du roman de Harriet Beecher-Stowe, Uncle Tom's Cabin (La Case de l'Oncle Tom, 1852), sur l'opinion publique s'explique par le contraste qu'il forme avec cet autoportrait des propriétaires d'esclaves sudistes. 


\section{Marx - Histoire universelle et questions contemporaines}

Karl Marx (1818-1883) et Friedrich Engels (1820-1895) n'ont pas développé de théorie autonome sur l'esclavage et l'économie antiques. Quand ils abordent le sujet, c'est généralement à l'occasion de brèves remarques, dans lesquelles l'Antiquité sert à illustrer des problématiques tout autres.

Pour Marx et Engels, les références à l'Antiquité étaient un patrimoine culturel évident. Marx avait, au lycée de Trèves en 1835, rédigé une dissertation de baccalauréat en latin sur l'heureuse ère augustéenne ${ }^{37}$, avant d'être, en 1841 , promu docteur en Philosophie à Jena (in absentia) avec une thèse sur la philosophie de la nature de Démocrite et d'Épicure ${ }^{38}$. Engels, qui avait abandonné le lycée un an avant le baccalauréat, n'avait accompli qu'une partie de cette formation classique ${ }^{39}$, mais il fut un autodidacte de génie. L'omniprésence, chez ces deux auteurs, d'exemples empruntés à l'Antiquité n'a en somme rien de surprenant - sauf peut-être pour ceux qui n'ont aucune idée du système éducatif allemand au XIXe siècle. Au fond, Marx et Engels ne firent que travailler sur la base de ce savoir scolaire. Leur exploitation de la littérature scientifique contemporaine sur l'Antiquité se limite en revanche à quelques travaux, en particulier ceux de Niebuhr et Mommsen pour l'histoire romaine, de Böckh et Grote pour l'histoire grecque 40 .

Les chercheurs qui, motivés ou non par des circonstances politiques conjoncturelles, ont tenté de reconstruire une théorie marxiste cohérente sur l'économie antique, ont dû le faire à partir de remarques éparses, en collationnant des passages d'œuvres scientifiques élaborées, comme le Capital ${ }^{41}$, des cahiers de notes, des pamphlets politiques, des écrits journalistiques (auxquels Marx se livrait surtout pour des motifs économiques ${ }^{42}$ ) et des lettres, quelles que soient leur valeur et leur date de rédaction

37 Version allemande : MEW, Suppl. I, 595-597, original : MEGA I, 1 (1975), 465-469 et, avec commentaire scientifique, chez BALDWIN (1988).

38 MEW, Suppl. I, 257-373.

39 Cf. les notes d'Engels remontant à ses années de lycée 1835/36, in MEGA IV, 1 (1976) $437 \mathrm{sv}$.

40 Références chez BACKHAUS (1974) 58 sv.

41 En raison du caractère scientifique de cette œuvre, la censure de l'empire tsariste autorisa, en 1872 , la publication d'une traduction russe et ce, malgré les opinions socialistes bien connues de l'auteur ; MEW 33, 492.

42 Cf. la remarque de Marx dans une lettre de 1853 : "Tartiner toujours du papier dans le journal m'ennuie. Ça me prend beaucoup de temps, je m'éparpille et en fin de compte ça n'est pas grand-chose. On a beau être aussi indépendant que l'on veut, on n'en est pas moins lié au journal et à son public, surtout quand on est payé comptant comme moi. Les travaux purement scientifiques, c'est tout à fait autre chose [...] »; MEW 28, 592 [trad. in Correspondance IV 26]. - Engels rédigea des articles d'histoire militaire pour Marx en tant que ghostwriter, afin de lui procurer des honoraires. 
respectives ${ }^{43}$. À propos de la réception de Marx par ses contemporains et par les différentes générations de ses commentateurs, il faut en outre noter que beaucoup de textes ont été éparpillés dans des publications difficilement accessibles, qui plus est, parfois, de manière partiellement anonyme, tandis que d'autres, totalement inédits, n'ont été publiés qu'ultérieurement, à partir des documents retrouvés après sa mort 44 .

Les lettres et articles de presse, si volontiers cités en raison de leurs formulations radicales, révèlent précisément que, dans bien des cas, Marx et Engels utilisèrent l'Antiquité dans une perspective contemporaine, lorsque qu'elle semblait offrir des parallèles avec le présent. Par exemple, l'un des plus longs développements de Marx sur les révoltes d'esclaves dans l'Antiquité a été écrit en mai 1860 pour le New York Daily Tribune. Il s'agit d'un résumé historique de la résistance sicilienne aux dominations étrangères, rédigé à l'occasion de la révolte qui venait juste d'éclater à Palerme contre le pouvoir des Bourbons. "Dans toute l'histoire de l'humanité, aucun pays ni aucun peuple n'a souffert aussi terriblement de l'esclavage, des conquêtes et de l'oppression étrangère que la Sicile et les Siciliens. » Plus loin, il évoque les guerres d'esclaves contre Carthage et Rome, la réduction massive en esclavage pratiquée par les Romains et l'importation d'esclaves supplémentaires. "Les Romains firent travailler le sol sicilien par d'innombrables équipes d'esclaves afin d'alimenter en blé sicilien les prolétaires démunis de la Ville Éternelle. " Le régime de terreur des gouverneurs romains était connu depuis Cicéron (dans le Discours contre Verrès). « Nulle part ailleurs, la cruauté romaine ne célébra de telles orgies. " On en arriva donc aux " plus terribles insurrections d'esclaves $[. .$.$] , pendant lesquelles la population locale et les esclaves importés firent$ souvent cause commune 45 ".

Une célèbre lettre de Marx à Engels, datant de la fin février 1861, éclaire le contexte de cet article : n'ayant pas eu le temps de lire les journaux sur la crise américaine qui s'aggravait, Marx avait lu « le soir, pour [se] détendre [...] les Guerres civiles romaines d'Appien, dans le texte grec original ". "Livre d'une grande valeur. [...] Spartacus y apparait comme le type le plus épatant de toute l'Antiquité ! Grand général (pas un Garibaldi), caractère noble, real reprensentative du prolétariat antique. Pompée : vrai salopard [...] ${ }^{46}$. " Cet enthousiasme pour Spartacus était donc déter-

43 Abstraction faite du cas particulier des instructions données par des partis communistes, c'est d'ailleurs un problème général avec les auteurs élevés au rang de "classiques "; il s'applique aussi, mutatis mutandis, à la " philologie weberienne".

44 On trouve une liste des publications de Marx dans l'article d'Engels à son sujet du Handwörterbuch der Staatswissenschaften (1892) ; MEW 22, 342 sv. [ENGELS, Heinrich Karl Marx].

46 MEW 30, 160 [trad. in Correspondance VI 285, trad. mod.] - Il est possible que Marx fanfaronne quelque peu et qu'en réalité, il ait lu Appien en traduction ; cela a été suggéré par l'étude d'AUDRING/SPERL (1997). 
miné par les circonstances, par l'observation des événements contemporains tant aux États-Unis qu'en Italie et en Sicile 47.

Marx et Engels n'ont certainement pas cru à une guerre antique pour abolir l'institution de l'esclavage. Il n'empêche que dans le Manifeste communiste (1848), la lutte des esclaves contre les hommes libres est comptée parmi les luttes de classes qui ont déterminé le cours de l'histoire ${ }^{48}$. "Dans le monde antique, le mouvement de la lutte des classes a surtout la forme d'un combat, toujours renouvelé, entre créanciers et débiteurs, et se termine à Rome par la défaite et la ruine du débiteur plébéien, remplacé par l'esclave ", lit-on dans le Capital de Marx ${ }^{49}$. Les esclaves étaient "sans droits ni volonté, dans l'impossibilité de se libérer, ainsi que la défaite de Spartacus l'a d'ailleurs montré ", note pour sa part Engels en 188250.

De même, dans les travaux systématiques, les exemples antiques ne sont souvent cités que pour illustrer des parallèles avec des phénomènes modernes, auxquels Marx s'intéresse au premier chef. Ainsi, dans le Capital, il cite longuement, dans une note de bas de page, la description faite par Appien de l'expropriation des petits propriétaires italiens par de grands propriétaires possédant des esclaves (avant même la lex Licinia de 367 av. J.-C.). Dans le passage correspondant - et donc dans les faits - il est question du développement de la propriété foncière en Angleterre au XVIIIe siècle, suite à l'introduction des clôtures. L'extrait d'Appien, qui ne sert ici qu'à compléter une longue citation concernant la situation anglaise, est d'ailleurs introduit par la formule : "On se rappelle les conflits de l'ancienne Rome ${ }^{51}$."

Une autre citation connue, à nouveau dans une note de bas de page du Capital, sur l'histoire de la propriété terrienne en tant qu'histoire "souterraine " de la

Le jugement dépréciatif sur Pompée tient au fait que celui-ci avait exagéré sa participation à la répression de la révolte de Spartacus (comme il l'avait fait auparavant pour son rôle dans la guerre contre Sertorius en Espagne). L'origine du jugement négatif de Marx sur Garibaldi dans cette lettre est plus obscur, car Engels avait, dans un article de 1860, vanté les capacités stratégiques de Garibaldi lors des campagnes de Sicile et d'Italie méridionale ; MEW 15, 60-62 et 150-158 [Engels, Garibaldi en Sicile, La Progression de Garibaldi et Garibaldi en Calabre].

48 MEW 4, 462 [MARX et ENGELS, Le Manifeste communiste].

49 MEW 23, 149 et sv. [MARX, Le Capital, trad. in Euvres I 678].

50 MEW 19, 302 [ENGELS, Bruno Bauer et le christianisme primitif]. - Voir aussi les propos de Lénine dans une conférence sur l'État de 1919 : «Les esclaves [...] se sont soulevés, se sont mutinés, ont déclenché des guerres civiles, mais jamais ils n’ont pu construire une majorité consciente du but à atteindre, des partis capables de diriger la lutte, jamais ils n'ont été à même de reconnaître clairement le but qu'ils poursuivaient et, même dans les moments les plus révolutionnaires de l'histoire, ils sont restés des pions dans les mains des classes dirigeantes "; LENIN (1961) 477.

51 Appien, Bellum civile I, 7, 30 sv. ; MEW 23, 754, note 211 [MARX, Le Capital, trad. in Euvres, I 1183]. 
République romaine ${ }^{52}$, fait en réalité partie d'une réponse à l'objection d'un spécialiste qui avait reproché à Marx d'avoir, dans sa Critique de l'économie politique, insisté, de manière partiale, sur l'importance du mode de production au détriment du rôle des conditions politiques dans le monde antique. Lorsque Marx affirme, dans la préface de la deuxième édition du 18 Brumaire de Louis Bonaparte (1869), que "dans l'ancienne Rome, la lutte des classes ne se déroulait qu'à l'intérieur d'une minorité privilégiée, entre les libres citoyens riches et les libres citoyens pauvres, alors que la grande masse productive de la population, les esclaves, ne servaient que de piédestal passif aux combattants 53 ", il espère écarter le " terme couramment employé aujourd'hui, particulièrement en Allemagne, de césarisme ", qui ne produit qu'une analogie historique superficielle 54 . On note, à d'autres endroits, un emploi métaphorique de la notion d'esclavage, celui-ci étant transposé à l'exploitation dont sont victimes les prolétaires modernes 55 .

Les références à l'esclavage revêtent une signification systématique lorsque Marx et Engels s'efforcent de couler l'Antiquité dans le moule d'une histoire universelle. Dans L'Idéologie allemande (1845/46), les deux auteurs établissent un lien entre la disparition de la propriété collective originelle au profit de la propriété privée et l'expansion romaine, à la suite de laquelle les petits propriétaires italiques, victimes d'une concentration de la propriété foncière, furent supplantés sur les grands domaines par des esclaves 56 . Les aspects juridiques de ce développement, par exemple la distinction entre propriété et possession, suivent pour le reste les préceptes de Niebuhr et Savigny 57. Ce texte n'a pas été publié ${ }^{58}$.

52 MEW 23, 96, note 33 [MARX, Le Capital.

53 Cf. la lettre de Marx à Engels du 8. 3. 1855 : «Il y a quelque temps, j’ai relu rapidement l'histoire romaine (ancienne) jusqu'à l'époque d'August[e]. L'histoire intérieure se ramène plainly [tout simplement] à la lutte de la petite propriété foncière contre la grande, avec, bien sûr, les particularités spécifiques qu'y introduit l'esclavage »; MEW 28, 439 [trad. in Correspondance, IV 211].

54 MEW 16, 359 [MARX, Le 18 Brumaire de Louis Bonaparte 12] - Pour les discussions au cours du XIXe siècle sur le césarisme, voir NIPPEL (2000a).

55 Par exemple dans le Manifeste communiste (MEW 4, 473); ou dans l'ouvrage d'Engels intitulé La Situation de la classe laborieuse en Angleterre (1845), dans lequel les prolétaires sont à plusieurs reprises désignés comme les « esclaves de la bourgeoisie " (MEW 2, 307, 310 et 350), et de plus, "de pires esclaves que les Noirs en Amérique, car ils sont plus étroitement contrôlés " (MEW 2, 400). MEW 3, 22 sv. [MARX, L'Idéologie allemande].

57 LEVINE (1987).

58 MARX (1859) : Du moment que la publication de L'Idéologie allemande n'a pu se faire comme prévu, " nous avons abandonné [...] le manuscrit à la critique rongeuse des souris "; MEW 13, 10 [MARX, Critique de l'économie politique]. La première édition date de 1932. 
Dans l'Anti-Dühring, qui date de la fin des années 1870, Engels expose sa thèse sur l'esclavage en tant qu'étape nécessaire sur la voie du progrès; cette vision n'était pas originale comme telle ${ }^{59}$, mais elle se voyait ici intégrée, en des termes particulièrement marquants, à une vision historique socialiste :

"Ce fut seulement l'esclavage qui rendit possible, sur une assez grande échelle, la division du travail entre agriculture et industrie et par suite, l'apogée du monde antique, l'hellénisme [Griechentum]. Sans esclavage, pas d'État grec, pas d'art et de sciences grecs; sans esclavage, pas d'Empire romain. Or, sans la base de l'hellénisme et de l'Empire romain, pas non plus d'Europe moderne. Nous ne devrions jamais oublier que toute notre évolution économique, politique et intellectuelle a pour condition préalable une situation dans laquelle l'esclavage était tout aussi nécessaire que généralement admis. Dans ce sens, nous avons le droit de dire : sans esclavage antique, pas de socialisme moderne.

Il ne coûte pas grand-chose de partir en guerre avec des formules générales contre l'esclavage et autres choses semblables, et de déverser sur une telle infamie un courroux moral supérieur $[\ldots .$.$] . Les anciennes communautés, là où elles ont subsisté, constituent depuis$ des millénaires la forme d'État la plus grossière, le despotisme oriental, depuis les Indes jusqu'en Russie. Ce n'est que là où elles sont dissoutes que les peuples ont progressé sur eux-mêmes, et leur premier progrès économique a consisté dans l'accroissement et le développement de la production au moyen du travail servile. [...] Si donc M. Dühring fronce le nez sur l'hellénisme parce qu'il était fondé sur l'esclavage, il aurait tout autant raison de reprocher aux Grecs de n'avoir pas eu de machines à vapeur et de télégraphe électrique ${ }^{60}$."

Lorsque dans le même contexte, Engels explique l'esclavage comme le résultat des guerres, il ajoute à une vision répandue, depuis les théories grecques 61 jusqu'aux défenseurs du droit des gens et du droit naturel, comme Hugo Grotius 62 et John Locke 63, en passant par les juristes romains ${ }^{64}$, selon laquelle l'esclavage reviendrait à renoncer à la mise à mort de l'ennemi fait prisonnier, une interprétation économique : " Jusquelà, on n'avait su que faire des prisonniers de guerre, on les avait donc tout simplement

59 On trouve par exemple des propos analogues chez Wilhelm von Humboldt, Böckh ou Nietzsche ; cf. MENZE (1987) ; DeIßLER (2000) 343 ; IDEM (2001).

60 MEW 20, 168 sv. [trad. in ENGELS, Anti-Dühring 213 sv.].

61 Aristote, Politique 1255 a5 sv.

62 De jure belli ac pacis (1625), Livre III, Chap. 7, $\$ 1$ = GROTIUs (1950), 480 sv.

63 Second Treatise of Government (1698), Chap. IV, $\$ 23=$ LOCKE (1988) 284, et à d'autres endroits. Pour la Caroline (future Caroline du Sud), Locke avait, en 1669 et en 1682, conçu une constitution pour une société esclavagiste; ARMITAGE (2004).

64 Florentinus, Digesta 1, 5, 4, 2 ; beaucoup d'autres passages dans WIELING (1999). Il s'agit, comme on le sait, de l'unique cas dans lequel les juristes romains voyaient un conflit entre droit des gens et droit naturel, ce qui ne les empêcha pas de confirmer la valeur de l'esclavage comme institution du ius gentium. 
abattus ; à une date plus reculée encore, on les avait mangés. Mais au niveau de l'“état économique" maintenant atteint, ils prenaient une valeur ; on leur laissa donc la vie et on se servit de leur travail. [...] L'esclavage était inventé ${ }^{65}$. " Cette conception de l'esclavage comme étape sur la voie de la victoire sur le cannibalisme et le sacrifice humain est caractéristique des théories évolutionnistes de l'histoire du genre humain ${ }^{66}$.

La référence au despotisme oriental nous amène naturellement à un problème auquel Marx et Engels se sont mesurés : existait-il un mode de production oriental ou asiatique spécifique, dans lequel, compte tenu de la combinaison de l'hégémonie absolue du chef sur toutes les terres et de la propriété collective en vigueur dans les campagnes, il n'y avait ni propriété privée ni esclavage ? Ces réflexions renvoyaient, d'une part, au débat sur la domination coloniale anglaise en Inde, d'autre part, à l'ancien modèle du despotisme asiatique, qui connaissait un regain d'actualité grâce aux récits de voyage en Inde du début des Temps modernes, comme celui de Bernier à la fin du XVII e siècle 67.

Marx utilise, dans l'avant-propos de sa Critique de l'économie politique (1859), cette célèbre formule : "Réduits à leurs grandes lignes, les modes de production asiatique, antique, féodal et bourgeois moderne apparaissent comme des étapes progressives de la formation économique de la société 68. "Ceci amène avant tout à se demander si le mode de production antique doit être clairement assimilé à une économie esclavagiste et s'il s'agit là d'une succession impérative de stades d'évolution de l'économie. Si l'on accepte l'hypothèse d'une succession à la fois inévitable et universelle, on se heurte alors au problème de savoir si - à l'encontre des hypothèses explicites de Marx au sujet de la stagnation asiatique - ce modèle s'applique aussi à l'Asie, ou si, au contraire, on doit postuler que le mode de production asiatique représente de la même façon un premier stade pour le développement économique européen. Sinon, il faut considérer ce stade comme une catégorie résiduelle, étrangère au schéma évolutionniste.

Les débats relatifs à ce schéma de développement, qui furent toujours lourdement influencés par des considérations politiques - la Chine doit-elle également passer par le

65 MEW 20, 167, cf. 169 [trad. in ENGELS, Anti-Dühring 212].

66 Cf. Auguste Comte dans les années 1830: «La guerre engendre l'esclavage, qui y trouve sa source et qui en est le premier correctif. L'esclavage remplaça l'anthropophagie ou l'immolation des prisonniers, dès que le vainqueur, maitrisant ses passions haineuses, comprit l'utilisé qu'il retirerait des services du vaincu en l'adjoignant à sa famille, à titre d'auxiliaire »; COMTE (1933) 208 [ = Sociologie 174] ; cf. aussi encore SCHMOLLER (1923) I, 364, à propos de la naissance de l'esclavage : "Là où dominait jusqu'alors le cannibalisme, où l'on considérait l'étranger comme n'ayant aucun droit, là où on l'aurait donc tué et mangé, c'était un grand progrès dans l'humanité et l'utilité économique de ne plus tuer les captifs ou les offrir en sacrifice. » (trad. in SCHMOLLER, Principes d'économie politique, II, 286).

68 MEW 13, 9 [MARX, Critique de l'économie politique, trad. in Euvres, I 273]. 
capitalisme pour atteindre le socialisme ou peut-on sauter cette étape ? - ont préoccupé nombre d'auteurs marxistes du XXe siècle ; leur investissement dans la scolastique MarxEngels semble complètement disproportionné par rapport aux résultats empiriques de cet effort.

Quant au thème de l'esclavage, l'affirmation de Marx, selon laquelle le passage d'un niveau social économiquement dépassé à un autre nécessitait une " époque de révolution sociale 69 ", était le prétexte pour assimiler les révoltes et les évasions d'esclaves de la fin du IIe et du Ier siècle av. J.-C., ainsi que les mouvements de révolte de la fin de l'Antiquité (Circoncellions en Afrique du Nord, Bagaudes en Gaule) à la révolution des esclaves (postulée par Staline) qui déboucha sur le féodalisme, même s'il fallait l'appeler une révolution " d'esclaves et de colons 70 ".

On peut, par ailleurs, douter du fait que Marx et Engels aient voulu par la suite maintenir ce concept de stades successifs (si toutefois il s'agissait de cela), alors qu’à partir de la fin des années 1860, divers travaux d'histoire agraire consacrés aux formes de propriétés collectives en Europe les amenèrent à remettre en cause la singularité de la situation asiatique et qu'ils revirent également en profondeur leur conception du développement de l'histoire universelle sous l'influence de Morgan et de Bachofen, qui avaient, pensait-on, ouvert des perspectives nouvelles sur la période antérieure à la tradition écrite ${ }^{71}$.

Les remarques de Marx et Engels sur les implications économiques de l'esclavage antique ne sont en général pas isolées ; rarement inscrites dans le contexte d'une présentation des époques de l'histoire universelle, elles sont le plus souvent liées à des prises de position dans le débat contemporain concernant l'esclavage. Quant au nombre d'esclaves, Marx s'est, dans ses publications, généralement exprimé par référence au contexte de la guerre civile américaine - plus il y avait d'esclaves par rapport à la population libre dans un État donné, plus la volonté de sécession y était forte ${ }^{72}$-, mais, sauf erreur de ma part, jamais en rapport direct avec l'Antiquité. Engels l'a, il est vrai, fait dans l'Origine de la famille, de la propriété privée et de l'État (1884), où il se fondait sur un évaluation traditionnelle remontant à Athénée. Il a d'ailleurs été âprement critiqué par la suite pour avoir naïvement reproduit une source douteuse, par exemple par Friedrich Vittinghoff 73 . Ce dernier néglige cependant le fait que, dans la recherche du XIXe siècle, cette question n'avait aucunement été tranchée de manière définitive en

69 MEW 13, 9 [MARX, Critique de l'économie politique, trad. in Euvres I 273].

70 VitTinghoff (1960) 124 sv. ; idem (1961) ; STAERman (1969) 27 sv. ; Heinen (1980) 273 sv. ; RASKOLNIKOFF (1990).

71 NipPEL (1990a) $112 \mathrm{sv.}$

72 Par exemple MEW 15, 342 [MARX, La Guerre civile aux États-Unis].

73 Vittinghoff (1960) 94. - Pour le contexte de l'essai de Vittinghoff, à savoir le « conflit Est-Ouest " au congrès international d'histoire de Stockholm en 1960, on devrait consulter non seulement les considérations polémiques et réductrices de FINLEY (1981a) 72 sv., mais aussi la présentation de ERDMANN (1987), en particulier 338 sv. et 430 sv. 
faveur des thèses de Hume. Il y avait, en effet, eu contre ce dernier une réplique contemporaine due à la plume de Robert Wallace ${ }^{74}$, qui avait préféré s'en tenir au chiffre traditionnel, une conception qui avait encore cours au XIXe siècle ${ }^{75}$. Engels parle non pas de 400 000, mais de 365000 esclaves ${ }^{76}$. Ce qui trahit, sans le moindre doute, l'origine de ses données, à savoir August Böckh, Die Staatshaushaltung der Athener, qui avait proposé cette légère révision à la baisse du chiffre " trop rond " d'Athénée ${ }^{77}$. Cette reprise de Böckh figure aussi dans l'un des cahiers de notes de Marx 78.

Marx et Engels suivent, sur presque tous les autres points, les arguments abolitionnistes qu'ils tirent de la littérature anti-esclavagiste relative au contexte américain, comme les écrits de Frederick Law Olmsted et John Elliot Cairnes, et ils les relient à des digressions sur l'esclavagisme antique. Cela a principalement été démontré par Wilhelm Backhaus, qui met également en avant le fait que, dans la littérature marxiste sur l'histoire de l'Antiquité soumise au dogme de la société esclavagiste, les citations isolées de Marx sont souvent présentées de manière telle que leur réel point d'ancrage, à savoir la situation américaine du XIX ${ }^{\mathrm{e}}$ siècle, est devenu méconnaissable ${ }^{79}$.

En 1852-55, Olmsted ${ }^{80}$, qui sera plus tard connu comme architecte paysagiste, avait, depuis les états sudistes, publié des bulletins réguliers dans le New York Times ${ }^{81}$. Il y adoptait le point de vue des propriétaires qui se vantaient d'assurer l'entretien à vie de leurs esclaves, quel qu'en soit le coût économique et qui sous-estimaient fortement les bénéfices tirés des plantations. Les prétendues découvertes d'Olmsted sur l'inefficacité du travail des esclaves reçurent leur consécration scientifique suprême par le truchement de l'économiste John Elliott Cairnes ${ }^{82}$. John Stuart Mill avait également fait

74 Wallace (1753), en particulier $280 \mathrm{sv}$.

75 DEIßLER (2000) 217 sv. Voir, outre les preuves avancées à cet endroit, BÜCHER (1922) 23.

76 MEW 21, 116 et 166 [ENGELS, L'Origine de la famille, de la propriété privée et de l'État].

77 BÖCKH (1817/1886) I, 47-49. - Dans le travail soviétique de LENCMAN (1966) 40 sv., il est admis qu'Engels avait repris le chiffre manifestement faux d'Athénée ; malgré la référence au calcul de Böckh (Ibid., 7), l'auteur ne s'aperçoit pas qu'Engels fait écho à l'évaluation figurant chez celui-là.

78 MEGA 7 (1983), 244-246.

79 BACKHAUS (1974). - Concernant le rapport de l'histoire ancienne soviétique avec Marx, on trouve déjà une remarque auto-critique dans STAERMAN (1969) 19 sv.

80 Olmsted (1822-1903) est connu pour avoir conçu le Central Park à New York, des parcs à Boston et dans une série d'autres villes, ainsi que les parcs naturels des Yosémites et des chutes du Niagara.

81 Des publications sous forme de livres ont suivi : OLMSTED (1856) ; IDEM (1861/1996). Sur Olmsted, voir Fogel/EngERMAN (1974) 170 sv. ; GERTEIS (1987) 157-163.

82 Cairnes (1823-1875) était originaire d'Irlande. Il fut professeur d'économie politique au Trinity College de Dublin (1856-1861), puis au Queen's College de Galway (1861-1866), où il donna des conférences qui servirent ensuite de base à son livre sur l'esclavage, et enfin à l'University College de Londres (1866-1872). 
l'éloge des articles d'Olmsted ${ }^{83}$. Dans le traité intitulé Slave Power (1862, 2e édition augmentée en 1863), Cairnes se fonde tout particulièrement sur les bulletins d'Olmsted. Il y résume les inconvénients économiques du travail servile en des formules marquantes :

"Slave labour is given reluctantly; it is unskilful ; it is wanting in versatility. It is given reluctantly, and consequently the industry of the slave can only be depended on so long as he is watched. [...] Secondly, slave labour is unskilful, and this, not only because the slave, having no interest in his work, has no inducement to exert his higher faculties, but because, from the ignorance to which he is of necessity condemned, he is incapable of doing so. [...] But further, slave labour is eminently defective in point of versatility. The difficulty of teaching the slave anything is so great, that the only chance of turning his labour to profit is, when he has once learned a lesson, to keep him to that lesson for life. Where slaves, therefore, are employed there can be no variety of production ${ }^{84}$."

Par ce livre, Cairnes contribua largement à rallier à la cause des États du Nord l'opinion publique anglaise 85 qui, malgré sa propre tradition abolitionniste, manifestait de la sympathie pour les États sudistes 86.

Dans le Capital, Marx établit, au moyen d'une longue citation de Cairnes, que l'exploitation extrême de la force de travail des esclaves équivalait finalement à un anéantissement du capital et qu'elle entraînait donc la nécessité d'un apport continu de

83 Principles of Political Economy 5 (1862), Livre II, Chap. 5 = Mill (2004) 252 : "The unproductiveness and wastefulness of the industrial system in the Slave States are instructively displayed in the valuable writings of Mr. Olmsted. " Olmsted avait dédicacé son ouvrage de 1861 à Mill ; Cairnes fit de même avec le sien.

84 CAIRnES (1863/2003) 44-47.

85 Cf. la lettre de Marx à Lassalle du 29. 5. 1861 : « Mais la presse anglaise officielle est naturellement pour les esclavagistes. Mais ce sont les mêmes types qui ont lassé le monde avec leur philanthropisme anti-commerce des esclaves. Mais coton, coton!"; MEW 30, 605 [trad. in Correspondance Marx-Lassalle 397] . Contre l'« affectation philanthropique " des aristocrates anglais, qui s'engageaient pour l'abolition de l'esclavage aux État-Unis, mais devaient leur richesse à l'exploitation de leur propre société, Marx s'était déjà insurgé dans un article pour le New York Daily Tribune 1853 ; MEW 8, 504 sv. - John Stuart Mill déclara, à propos de l'attitude des classes dirigeantes anglaises au début de la guerre civile américaine : "The feelings of the present generation of Englishmen [...] have fallen behind the positive acts of the generation which preceded them "; ce serait un "lasting blot in English history"; MiLL (2004) 254.

86 Cet impact sur l'opinion publique anglaise fut déjà constaté ou prévu dans des recensions (anonymes) qui suivirent la parution du livre ; par exemple, dans Spectator 1862, 476, et dans Westminster Review 1862, 303 sv. - Sur Cairnes et son influence, voir MILLER (1963/64) ; Den Hollander (1967) ; Fogel/Engerman (1974) 181 sv. ; BackHaus (1975) ; M. M. SMITH (2003). 
main-d'œuvre par le biais de la traite ${ }^{87}$. Marx n'était toutefois pas certain que ce raisonnement s'appliquât aussi, plus généralement, à l'Antiquité. Dans le même livre, il se réfere à un passage de Diodore selon lequel certains esclaves auraient, dans les mines d'or d'Égypte, d'Éthiopie et d'Arabie, travaillé jusqu'à la mort, mais il ajoute aussi qu'il s'agit d'un cas exceptionnel dans l'Antiquité 88 .

Qu'il y ait bien eu, même dans l'Antiquité, la nécessité d'un afflux continu d'esclaves, venant d'un marché nourri par la guerre et les razzias, Marx le démontre a fortiori par l'exemple des États sudistes, dans lesquels l'élevage d'esclaves n'était pas suffisant ${ }^{89}$. Marx connaissait les chiffres du recensement américain, qui révélaient une augmentation spectaculaire de la population d'esclaves dans les États du Sud jusqu'à la guerre civile (nous y reviendrons) ; il croyait toutefois que ce phénomène avait longtemps reposé sur les importations d'esclaves en provenance d'Afrique 90 .

La théorie plus tardive du "sabotage ", autrement dit le fait que l'on ne pouvait confier aux esclaves que des outils primitifs ou des animaux peu exigeants parce qu'ils détruisaient les outils de travail plus efficaces, provient également de la discussion américaine, en l'occurrence d'Olmsted, dont le texte n'avait vraisemblablement été porté à la connaissance de Marx que de seconde main, par l'intermédiaire de Cairnes ${ }^{91}$. Nous ignorons totalement si Marx voulait projeter sur l'Antiquité cette hypothèse telle quelle 92 .

À divers endroits, Marx et Engels affirment que l'une des conséquences du travail des esclaves avait été de déclasser socialement le travail salarié, les citoyens libres refusant désormais une activité considérée comme servile. Ceci avait finalement constitué l'obstacle majeur au développement de toute économie fondée sur le travail servile 93. Quelques sources antiques avaient pu être trouvées pour étayer cette thèse ${ }^{94}$; remarquons tout de même que la plèbe romaine est, en tant que sous-prolétariat ${ }^{95}$, mise en parallèle avec les poor ou les mean whites, c'est-à-dire avec les milieux blancs les plus

87 MEW 23, 281 sv. [MARX, Le Capital] = CAIRnEs (1863/2003) $122 \mathrm{sv.}$

88 Diodore III, 13. - MEW 23, 250 [Marx, Le Capital].

89 MEW 24, 474 sv. [MARX, Le Capital].

90 MEW 23, $467 ; 24,475$ [MARX, Le Capital].

91 MEW 23, 210, note 17 [MARX, Le Capital] ; BACKHAUS (1974) 74.

92 Cette hypothèse est (entre autres) clairement transposée dans l'Antiquité chez KAUTSKY (1908) 40 sv. ; IDEM (1911) 722.

93 MEW 20, 585 sv. [ENGELS, Matériaux pour l'Anti-Dühring].

94 Voir STE Croix (1981) 181, 185 et 199-201, qui évoque toutefois aussi la mentalité des classes inférieures blanches des États sudistes, qui refusent " to work like a nigger ».

95 Accessoirement dans MEW 3, 23 [MARX, L'Idéologie allemande]. - D’une manière générale, voir, pour cette catégorie en rapport avec le XIXe siècle, MEW 4, 472 [MARX, Le Manifeste communiste] ; 7, 26 [MARX, La Lutte des classes en France] ; 8, 160 sv. [MARX, Le 18 Brumaire de Louis Bonaparte]. 
défavorisés des États esclavagistes américains ${ }^{96}$. L'existence de cette couche sociale également appelée white trash, qui n'acceptait aucun travail normalement presté par des esclaves, était par exemple décrite par Cairnes comme une "invariable outgrowth of negro slavery wherever it has raised its head in modern times 97 ".

En résumé, Marx ne s'est intéressé que de manière marginale à l'esclavage et à l'économie antiques. Ce qui lui tenait à cœur, c'était une théorie du capitalisme moderne, dont la caractéristique déterminante était l'utilisation du travail formellement libre. Le modèle capitaliste se définit notamment par le fait que l'« ouvrier salarié libre vend sa force de travail au capital " et que "l'ouvrier lui-même apparait simplement comme vendeur de marchandises, par conséquent comme travailleur salarié libre, le travail n'apparaissant plus que sous la forme de travail salarié 98 ".

Les distinctions entre diverses formes pré-capitalistes, l'intervention de la contrainte extra-économique, que ce soit sous forme d'esclavage ou d'autres formes de dépendance ou de travail forcé, ne l'intéressent que dans certains contextes d'argumentation ${ }^{99}$. L'intérêt spécifique pour l'esclavage dans les États sudistes (mis à part son importance pour la guerre civile) naît, d'un point de vue théorique, avant tout du fait qu'il y voit une "anomalie à l'intérieur d'un marché mondial reposant sur le travail libre 100 ». Ses références à l'esclavage antique sont en quelque sorte les sous-produits d'un intérêt essentiellement tourné vers le présent.

96 MEW 15, 337 [MARX, La Guerre civile nord-américaine] ; 19, 111 sv. ; 21, 144 [ENGELS, L'Origine de la famille, de la propriété privée et de l'État] ; 22, 463 [ENGELS, Heinrich Karl Marx]. - Marx voyait dans l'existence et la mentalité de cette classe la cause de l'absence de développement, aux États-Unis, d'un mouvement ouvrier, et il espérait à ce sujet qu'il en irait autrement après la guerre civile et l'émancipation des esclaves ; MEW 16, 19 (Vœux adressés par l'Association ouvrière internationale au président Abraham Lincoln, décembre 1864); MEW 23, 318 [MARX, Le Capital].

97 CAIRnes (1863/2003) 82 sv. - Cf. Olmsted (1861/1996) 64-66 : « No white man would ever do certain kinds of work [...] ; and if you should ask a white man you have hired, to do such things, he would get mad and tell you he wasn't a nigger. [...] The poor white people [...] are said to be extremely ignorant and immoral, as well as indolent and unambitious. [...] They seem [...], more than any other portion of the community, to hate and despise the negroes. "Ibid., 87 : "The employment of whites in duties upon which slaves are ordinarily employed is felt to be not only humiliating to the whites employed, but also to the employer. "

98 MEW 23, 354 ; 25, 886 [trad. in Le Capital, XIV 211 (trad. mod.)].

99 Voir les Fondements de la critique de l'économie politique, en particulier le chapitre sur les "Formes qui ont précédé la production capitaliste" ; MEW 42, 383-421. Ce texte de Marx de 1857/58 n'a d'ailleurs été publié qu'en 1939, puis en 1953, à partir des archives.

100 MEW 42, 420 [MARX, Les Fondements de la critique de l'économie politique]. 


\section{Mommsen - Capitalisme et esclavage}

L'importance accordée, dans une perspective d'histoire universelle, à la singularité du capitalisme moderne conditionne aussi, chez Marx, la polémique dirigée contre la façon dont Mommsen traite du capitalisme dans son Histoire romaine. Le fait que Mommsen emploie le terme "capitalisme " dès qu'il a affaire à une économie monétaire montre qu'il n'a aucune notion du concept moderne de capital, et qu'il anéantit la différence essentielle entre sociétés antiques et sociétés modernes, lit-on à plusieurs endroits du Capital 101. Mais en dépit de cette critique fondamentale, l'image que Marx et Engels se sont faite de l'histoire romaine est influencée à plusieurs égards par Mommsen 102.

Même s'il procède de manière plutôt impressionniste, Mommsen établit lui aussi, dans son Histoire romaine, des parallèles constants entre esclavage antique et esclavage moderne nord-américain. Son œuvre date des années 1850, c'est-à-dire d'avant la guerre civile américaine. Que les deux situations puissent être comparées semble aller de soi à ses yeux, même s'il suppose que l'économie esclavagiste des États sudistes n'a pas encore atteint la même ampleur qu'à Rome. L'économie des latifundia qui caractérise l'Italie à partir du IIe siècle av. J.-C. est comparable à l'économie des plantations américaines ; l'exploitation des esclaves, qui « accordait peu de prix à la vie et à la reproduction ", exigeait sans cesse de nouvelles guerres, et en outre, comme celles-ci ne suffisaient pas à satisfaire la demande du marché, une chasse aux esclaves continuelle. «Le pays nègre, pour les Romains, c'était l'Asie occidentale 103. » Il suffisait selon lui "d'un coup d'œil jeté sur la condition de ces plus infimes [...] pour affirmer [...] que les nègres n' [avaient] eu qu'une goutte d'eau du calice à boire, auprès des maux soufferts par les esclaves romains 104 ». Mais comme les révoltes l'ont montré, l'esclavage représentait pour l'État romain un danger, auquel on aurait dû se préparer bien plus efficacement. Seuls les anxieux ou les " trembleurs politiques " prédisaient la " destruction de l'ordre social, par l'effet des révoltes serviles ou par les insurrections des prolétaires 105 ».

101 MEW 23, 182, note 39; 25, 339, note 46 ; 795 avec note 43 [MARX, Le Capital.

102 Pour la lecture et la critique de Mommsen par Engels, voir aussi les lettres de ce dernier à Marx du 26.3.1858 et à Karl Kautsky du 20.10.1884; MEW 29, 308 ; 36, 224 ; voir aussi MEW 21, 119-123 [ENGELS, L'Origine de la famille, de la propriété privée et de l'État]. Th. MOMMSEN (1902) II, 75 [trad. in Histoire romaine I 762].

104 Ibid., II, 77 [trad. in Histoire romaine I 763] - Mommsen suit ici, consciemment ou non, un schéma d'argumentation allant de Thomas Jefferson aux défenseurs univoques de l'esclavage dans les États sudistes, selon lequel leurs conditions se distinguaient positivement de celles des esclaves antiques alors que les abolitionnistes affirmaient que leur propre système d'esclavage était le pire qui ait existé dans l'histoire mondiale : voir l'affirmation de D. B. Davis, citée par WeILeR (2003) 100 ; voir aussi BaCKHAUs (1975) 544 sv. Th. Mommsen (1902) II, 79 [trad. in Histoire romaine I 765]. 
Comme le nécessaire " régiment de la terreur » faisait défaut, il fallut avoir recours à des mesures militaires et à des exécutions, si bien qu'il ne fut plus possible, d'" épargner encore le capital 106 ».

L'effet destructeur de l'esclavage causé par l'immense fossé séparant les pauvres des riches du point de vue des mours, des liens familiaux et du respect de la propriété est toujours le même : «Dans tout État à esclaves, aussitôt que s'établit et règne le capital, il ravage et détruit le monde sorti splendide de la main de Dieu ${ }^{107}$. " C'est avant tout par le biais de l'économie esclavagiste que le capitalisme montre ses conséquences les plus fatales : "Quels qu'aient été les torts de lèse-nation et de lèse-civilisation que l'on a pu, de nos jours, jeter à la face du système capitaliste, ces torts ne sont rien, comparés aux crimes des anciens États capitalistes, de même que l'homme libre, si pauvre qu'il soit, reste bien au-dessus des esclaves. Que la semence de dragon jaillisse à nouveau sur les terres d'Amérique du Nord, et le monde verra fleurir à nouveau de semblables récoltes 108 ! " Cette dernière invocation n'est pas sans rappeler la peur de Jefferson face au loup " qu'on ne peut plus tenir par les oreilles ». Mommsen prophétisait, fût-ce de façon un peu cryptée ${ }^{109}$, un régime césarien pour les États sudistes si le niveau d'esclavage venait à y égaler le niveau romain (ce qui était en fait le cas depuis longtemps).

"L'ouvvre de César était nécessaire et salutaire [...] parce qu’au sein du système antique, assis sur l'esclavage [...] la monarchie militaire absolue était devenue la clé de voûte indispensable, logique, et qu'elle était enfin le moindre des maux. Vienne le jour où l'aristocratie à esclaves des Virginies et des Carolines se sera, dans cette voie, avancée aussi loin que la société sœur de la Rome de Sylla, le césarisme y surgira, encore une fois légitimé par l'histoire. À l'inaugurer ailleurs et dans de tout autres conditions sociales, il n'y a que parodie et usurpation ${ }^{110}$."

\section{Weber - L'esclavage dans une perspective d'histoire universelle}

Mommsen représente, d'une certaine façon, une passerelle entre Marx et Weber. Max Weber (1864-1920) s'était, à l'instar de Marx, donné comme objectif de mettre en lumière la spécificité de l'Occident moderne et en particulier de son capitalisme rationnel. Mais contrairement à Marx, il le fit sur la base de recherches approfondies sur les phases pré-modernes de l'Europe et, dans ses travaux ultérieurs de sociologie des reli-

106 Ibid., II, 79 [trad. in Histoire romaine I 765].

107 Ibid., III, 532 [trad. in Histoire romaine II 414].

108 Ibid., III, 533 [trad. in Histoire romaine II 414 (trad. mod.)].

109 Voir l'incompréhension de MEYER (1924) 210, note 4. [p. 371].

110 Th. Mommsen (1902) III, 477 sv. [trad. in Histoire romaine II 371]. 
gions, en mettant l'accent sur les conditions sociales, en particulier religieuses, qui ont, en Asie, contrarié cette évolution vers le capitalisme rationnel ${ }^{111}$.

Weber a souvent été désigné par la postérité comme un "Marx bourgeois ». Ce surnom correspond bien à ses propres conceptions dans la mesure où il considère l'analyse sociale marxiste comme un défi intellectuel fondamental, mais il juge scientifiquement irrecevable la démarche philosophico-historique consistant à postuler des nécessités historiques. Weber a exposé sa position dans un essai programmatique rédigé à l'occasion de son entrée en fonction comme co-directeur de la revue Archiv für Sozialwissenschaft und Sozialpolitik, en 1904.

"Encore que nous ne partagions aucunement le préjugé désuet suivant lequel la totalité des manifestations d'ordre culturel se laisserait déduire comme produit ou comme fonction de constellations d'intérêts "matériels", nous croyons cependant pour notre part que l'analyse des phénomènes sociaux et des événements culturels, sous le point de vue spécial de leur conditionnalité [Bedingheit] et de leur portée économique, a été un principe scientifique d'une fécondité créatrice et qu'elle le restera sans doute dans l'avenir le plus lointain, à condition de l'employer avec prudence et de la débarrasser de toute prévention dogmatique. Certes, la soi-disant "conception matérialiste de l'histoire", considérée comme une "conception du monde" ou comme le dénominateur commun [Generalnenner] de l'explication causale de la réalité historique, doit être rejeté de la façon la plus catégorique ; néanmoins, le souci d'une interprétation économique de l'histoire est un but essentiel de notre Revue ${ }^{112}$."

Peu avant sa mort en 1920 il déclare :

"L'honnêteté d'un intellectuel d'aujourd'hui, et avant tout d'un philosophe d'aujourd'hui, peut se mesurer à son attitude face à Nietzsche et à Marx. Celui qui ne concède pas qu'il n'aurait pu mener des parties essentielles de son œuvre sans le travail que ces deux-là ont accompli escroque lui-même et les autres. Le monde dans lequel nous existons nousmêmes intellectuellement est en bonne partie un monde formé par Marx et Nietzsche ${ }^{113}$."

Dans un discours sur le socialisme prononcé devant des officiers à Vienne, en juin 1918, Weber qualifie même le Manifeste communiste de contribution scientifique de premier plan ; certes, il en réfute de nombreuses thèses, mais il les considère comme des erreurs «brillantes », qui ont été fertiles pour la science. Il se montre par ailleurs impressionné par le fait que le texte se soit abstenu de toute moralisation sur « tout ce que le

111 Voir, pour un aperçu de l'œuvre de Weber dans le domaine de l'histoire antique, NIPPEL (2000b) qui contient des références à la littérature secondaire.

112 GAWL 166 et sv. [trad. in Essais sur la théorie de la science 147].

113 Reproduit chez BAUMGarten (1964) 554 et sv., cité également par HenNis (1996) 181. 
monde a de mauvais et de bas 114 ». Weber fut, on le sait, toute sa vie durant partisan d'une formulation aussi brutale que possible des réalités sociales et, en ce sens, proche de Marx. Dans ses publications scientifiques, les références explicites à ce dernier sont cependant rares, et ne sont pas spécialement productives ${ }^{115}$. Concernant la " conception matérialiste de l'histoire ou vision du monde ", il se réfere surtout aux œuvres de Karl Kautsky et du jeune Werner Sombart.

Weber n'a été élevé au rang de "père fondateur de la sociologie " qu’à titre posthume ; à son époque, cette branche n'existait pas encore en tant que discipline académique reconnue. Weber a été formé comme scientifique dans les domaines de l'économie politique historique et de l'histoire du droit ${ }^{116}$. Cela impliquait, dans son cas, un intérêt particulier, mais non pas exclusif, pour l'Antiquité. Dans ses travaux s'y rapportant, Weber se fonde d'abord sur Mommsen ${ }^{117}$; l'élargissement ultérieur de sa perspective, au-delà de Rome, à l'ensemble de l'Antiquité méditerranéenne et procheorientale est redevable à l'étude des travaux d'Eduard Meyer 118 ainsi qu'à de nombreux autres travaux scientifiques sur l'Antiquité.

Les détails techniques (complexes) de sa thèse d'habilitation en droit romain et en droit commercial, intitulé " Histoire agraire de Rome » (1891), ne nous intéressent pas dans le cadre de cet exposé ; notons seulement que ce travail doit l'essentiel de sa configuration générale aux thèses proposées par Mommsen dans l'Histoire romaine. Weber suit, par exemple, le raisonnement de ce dernier lorsqu'il affirme que Rome a développé sur l'ager publicus une forme de capitalisme agraire, d'après lui le capitalisme le plus débridé qui ait jamais existé dans ce domaine au cours de l'histoire 119 .

Weber a toujours considéré le concept de capitalisme antique comme utile. Dans l'introduction théorique de son long article "Agrarverhältnisse im Altertum" paru dans le Handwörterbuch der Staatswissenschaften (1909), il définit également sa position vis-à-vis de la controverse Bücher-Meyer ${ }^{120}$. Il refuse l'interprétation moderniste de l'économie antique proposée par Meyer, tout en continuant à considérer le capitalisme comme une catégorie pertinente dans l'histoire universelle. La limitation de la notion de capitalisme à la «caractéristique sociale " de l'« exploitation du travail d'autrui par l'intermédiaire

114 MWG I/15, 616 [trad. in Euvres politiques 474].

115 GAWL 163 et 246 ; GASS 450 ; GARS II, 109 ; WuG 61 et 179.

116 Voir récemment MARRA (2002).

117 Les relations entre les familles Mommsen et Weber, ainsi que l'estime de Mommsen pour le jeune Max Weber, qu'il avait déclaré son successeur présumé à l'occasion de la soutenance de thèse de ce dernier en 1889, interviennent également ; DEININGER in MWG I/2, 57 et sv. ; après l'habilitation de Weber, Mommsen se demanda, dit-on, s'il recommanderait celui-ci pour une chaire d'histoire antique ou pour une chaire de droit romain ; HONIGSHEIM (1963) 205 et 221.

118 Sur la relation Weber-Meyer, voir NiPPEL (1991) ; IDEM (1996).

119 MWG I/2, 216.

120 Cf. MAZZA (1985) ; SCHNEIDER (1990) ; IDEM (1999). 
d'un contrat avec le travailleur "libre" " n'est pas convaincante à ses yeux puisqu'il s'agit d'un critère s'appliquant uniquement à l'époque moderne ${ }^{121}$ - il y a là (quelle que soit l'appréciation que l'on donne de cette prise de position), un différend fondamental avec Marx et ses adeptes, que Weber ne nomme pas, mais auxquels il pense certainement.

En s'en tenant à une notion de capitalisme qui transcende les époques et les cultures, Weber croyait précisément pouvoir mieux mettre en évidence les différences entre ces diverses formes de capitalisme. Dans ses écrits ultérieurs de sociologie des religions, il mentionne des "formes spécifiques du capitalisme antique, extra-européen et du capitalisme qui a précédé le développement bourgeois moderne ", qu'il qualifie de capitalisme colonial, de capitalisme de fournisseurs de l'État, de capitalisme de fermiers d'impôts, de droits de douane et de monopoles étatiques ${ }^{122}$.

Weber a naturellement montré à maintes reprises l'importance du travail libre et il ne songeait nullement à gommer la différence entre économie pré-moderne et moderne. Cependant, en fonction de l'orientation de son argumentation, il a souligné tantôt les parallèles, tantôt les différences ${ }^{123}$. Dans ses prises de position sur la question agraire à l'est de l'Elbe, il a, dans les années 1890, mis en évidence les points communs en insistant sur les possibilités limitées de couvrir les besoins saisonniers fluctuants de main-d'œuvre agricole. Lorsqu'il attribue aux saisonniers polonais un niveau de culture servile 124 , ce qui invalide, constate-t-il, la règle traditionnelle (de Columelle) qui veut que la culture des céréales soit l'affaire d'ouvriers culturellement développés ${ }^{125}$, cela correspond à son argumentation politique : sur la nécessité de renforcer la germanité des régions frontalières orientales et d'assurer ainsi, également, une base de recrutement pour l'armée ${ }^{126}$.

Dans un texte tiré d'une conférence fribourgeoise sur les " causes sociales du déclin de la civilisation antique ", en 1896 , Weber déclare en revanche, de manière un tant soit peu provocatrice, que ce thème est d'un intérêt exclusivement historique : «[...] un prolétaire d'aujourd'hui et un esclave antique se comprendraient aussi mal qu'un Européen et un Chinois ${ }^{127}$. "Quand on lit, au même endroit, qu'à ce sujet, on ne peut appliquer au lecteur l'aphorisme de te narratur fabula, cela pourrait bien être une attaque directe contre Marx, qui avait utilisé cette citation d'Horace comme

121 GASWG 15 [trad. in Économie et société dans l'Antiquité 101].

122 GARS III, 359 [trad. in Le judaïsme antique 453] ; cf. GARS I, 49, note 2 [trad. in L'Éthique Protestante 44] ; WuG 368 sv. [trad. in Economie et société, coll. Agora, vol. 2, 385 sv.].

123 Concernant le rapport entre les recherches de Weber en histoire ancienne et en économie politique dans sa première phase d'activité, voir NIPPEL (1990b) ; IDEM (1994).

124 GASWG 482 (= "Die ländliche Arbeitsverfassung") ; voir aussi MWG I/4, 397 et 447 ; GARS I, 27, note 3. [WEBER, L'Éthique protestante].

125 MWG I/3, 228 ; cf. MWG I/2, 299, note 7, avec référence à Columelle I, 7, 6.

126 Ainsi surtout dans sa leçon inaugurale de Fribourg en 1895 ; GPS 1-15. [Weber, Euvres politiques].

127 GASWG 291 [trad. in Économie et société dans l'Antiquité 65]. 
" devise " dans le premier volume du Capital ${ }^{128}$. Dans le même essai, on trouve par ailleurs des allusions claires au vocabulaire marxiste. On lit par exemple : «Le propriétaire d'esclaves devint ainsi le support économique de la civilisation antique, et l'organisation du travail des esclaves constitua le fondement [Unterbau] indispensable de la société romaine ${ }^{129}$. " La thèse générale de Weber, selon laquelle la fin des conquêtes romaines et les charges que l'appareil étatique de plus en plus bureaucratique et l'armée faisaient peser sur la société avaient rendu l'esclavage économiquement obsolète, ne se distingue pas en substance des déclarations analogues d'Engels 130 .

Les explications de Weber sur l'Antiquité se concentrent sur les limites du développement d'un capitalisme antique qui, reposant sur l'exploitation des opportunités de gain fournies par des opérations politico-militaires, aurait été, sous l'Empire, étouffé par les structures bureaucratiques.

Depuis l'Histoire agraire romaine jusqu'aux Agrarverhältnisse im Altertum en passant par l'essai sur le déclin de la civilisation antique, l'intérêt de Weber glisse de l'analyse des changements structurels dans l'économie de l'empire romain à une reconstruction idéal-typique des spécificités de l'économie antique, y compris de celles des grandes civilisations du Proche-Orient. À cet égard, il ne se limite pas - comme Marx et Mommsen - à l'examen du travail servile dans l'agriculture, mais il traite aussi des exploitations industrielles [Gewerbebetriebe]. Dans ces deux domaines, il relève toutefois dans l'esclavage des faiblesses fondamentales au niveau de la rentabilité par rapport à une organisation du travail basée sur un contrat formellement libre.

Selon Weber, la culture antique repose en tout cas, à l'apogée des cités libres, sur le travail servile, même si dans l'article de 1909, il concède que, dans ses travaux précédents, dans le souci de "faire ressortir les caractères spécifiques de l'économie de l'Antiquité, au nombre desquels figure incontestablement le travail servile " [...], il avait sous-estimé « l'importance quantitative du travail libre ${ }^{131}$ ».

Parmi les caractéristiques économiques de l'usage capitaliste de la possession d'esclaves, Weber signale le besoin accru de capitaux destinés à l'achat de ceux-ci ; les époques de non-activité des esclaves et, de ce fait, leur " entretien pendant plusieurs mois 132 ", qui faisaient que "ce capital dévore continuellement des fonds supplémentaires 133 "; à Rome, la nécessité, en cas de pénurie de main-d'œuvre libre, d'occuper

128 Horace, Satires I, 1, 69 et sv. Marx cite une autre fois ce passage dans le texte: MEW 23, 282 [MARX, Le Capital] ; voir BruHns (1998) 58 sv. - Dans la Römische Agrargeschichte, Weber fait une allusion ironique au best-seller d'August Bebel, La Femme et le socialisme; MWG I/2, 349 ; voir aussi MWG I/4, 129.

129 GASWG, 296 [trad. in Économie et société dans l'Antiquité 69].

130 MEW 21, 143 sv. [ENGELS, L'Origine de la famille, de la propriété privée et de l'État].

131 GASWG, p. 11 [trad. in Économie et société dans l'Antiquité 97].

132 MWG I/2, 313.

133 GASWG 18 ; cf. 244 [trad. in Économie et société dans l'Antiquité 104 (trad. mod.)]. 
des esclaves en quantité suffisante pour répondre aux besoins les plus aigus, menait à une tendance nouvelle que l'on observe sous l'Empire. On couvrait, en effet, les besoins en biens industriels par l'auto-production, ce qui minait à nouveau les bases du développement d'une économie de marché ; le taux de mortalité des esclaves mettait en péril le capital investi ; les variations des prix des esclaves entraînaient le risque de dévaluation du capital, ainsi que l'absence d'un calcul fixe du coût du travail ; la pleine exploitation de la main d'œuvre n'était possible que si les esclaves vivaient, sans famille, dans un système de caserne ; si on les en laissait sortir, la valeur économique de l'esclavage régressait ; l'élevage d'esclaves était, quant à lui, assez improbable, dans la mesure où l'on dépendait d'un flux constant d'esclaves venant de l'extérieur et où le marché des esclaves dépendait lui-même des guerres, qui fonctionnaient aussi comme une chasse à l'homme :

" La caserne d'esclaves était incapable de se reproduire elle-même, elle devait être complétée par l'achat continu d'esclaves supplémentaires [...]. L'entreprise esclavagiste antique est dévoreuse d'hommes, comme le haut fourneau moderne de charbon. Le marché d'esclaves et son approvisionnement régulier et suffisant en matériel humain est une condition indispensable de la caserne d'esclaves destinée à produire pour le marché. [...] Ainsi, une telle entreprise est tributaire d'un apport régulier d'hommes sur le marché du travail. Que se passerait-il s'il venait jamais à manquer ? Cela ne laisserait pas de retentir sur la caserne d'esclaves, de même que l'épuisement des mines de charbon retentirait sur les hauts fourneaux ${ }^{134}$."

Cela signifie concrètement, pour le développement romain, que la fin des guerres d'expansion sous l'Empire a déterminé la tendance à abandonner la caserne d'esclaves 135 et à permettre à ceux-ci de fonder une famille, si bien que leur statut se rapprochait de celui des colons. Dans l'ensemble, ce sont l'instabilité constitutive du capital existant, les limites de l'exploitation du travail servile, l'impossibilité d'un calcul rigoureux lorsqu'on utilise du travail servile, le manque d'esprit d'entreprise des élites, qui traitent leurs esclaves comme des fonds de rente, ainsi que le manque de stimulants aux progrès techniques et à la hausse de qualité - due au manque d'intérêt personnel de la main-d'œuvre servile d'une part, à la disponibilité générale de main-d'œuvre non libre de l'autre, qui auraient été responsables, avec divers autres facteurs, de la capacité limitée de développement économique de la Rome antique par rapport à celle de l'économie moderne.

Bien que Weber ait eu un regard bien plus ample et plus précis sur l'Antiquité et qu'il ait disposé de sources et de connaissances bibliographiques bien plus larges sur ce

134 GASWG 298 sv. [trad. in Économie et société dans l'Antiquité 71].

135 L'image de la caserne d'esclaves se fonde d'une part sur Columelle, de l'autre sur un parallèle avec le système militaire prussien (MWG I/2, 346 ; GASWG 243 sv. et 297 sv.); elle semble lui avoir été inspirée par le souvenir de son service militaire. 
sujet que Marx, il existe des parallèles frappants entre les deux conceptions, notamment dans la mesure où ils se concentrent sur l'aspect purement économique de l'esclavage et font l'impasse sur les considérations éthiques.

Weber se fonde certainement, dans ses considérations sur l'esclavage antique, sur Appien et les écrivains agraires romains, ainsi que sur Xénophon, Démosthène, Lysias et d'autres. Il faut toutefois se demander s'il ne part pas lui aussi d'une certaine image de l'esclavage dans l'Antiquité, qui serait imprégnée des situations modernes et du discours sur l'esclavage des États sudistes nord-américains. À ma connaissance, l'immense littérature secondaire concernant Weber ne comprend, hélas, aucune étude sur ce point ${ }^{136}$. La question n'est pas simple à résoudre car, en général, Weber ne se réfere pas, ou bien il le fait uniquement de manière très sélective, aux ouvrages qu'il utilise tout à l'inverse de Marx, qui nourrit ses écrits d'économie politique de longues citations de la littérature spécialisée, révélant ainsi les sources de sa connaissance ${ }^{137}$.

Il est pourtant possible de rassembler quelques indices en faveur d'un recours par Weber au discours esclavagiste du XIXe siècle. Par exemple, il est frappant de voir qu'il parle de plantations à propos des grandes entreprises agricoles antiques exploitées par des esclaves ${ }^{138}$, qu'il utilise l'image « des esclaves de plantations enchaînés et travaillant sous le fouet 139 ", et qu'il insiste sur le fait qu' "on n'a encore jamais réussi à faire produire avec du travail non libre pour le marché $[\ldots]^{140}$ », en tout cas pas de manière durable.

Dans Économie et société, Weber parle explicitement d'un type similaire d' "économie de plantation basée sur le travail non libre " pour Rome et les États sudistes nordaméricains ${ }^{141}$. Il considère comme un fait avéré que ce système n'est rentable que s'il est possible de nourrir les esclaves à très bon prix, dans le cadre d'un marché d'esclaves

136 Entre-temps beaucoup d'ouvrages importants ont paru sur le thème "Weber et l'Antiquité "; nous ne les énumérons pas dans la bibliographie afin de ne pas l'alourdir, et nous nous contentons de renvoyer à certains de nos propres travaux où l'on trouvera de nombreuses références bibliographiques.

137 Voir Engels dans l'avant-propos de l'édition anglaise du Capital en 1886 ; MEW 23, 38 : "Ces citations dotent [...] le texte d'un commentaire courant emprunté à l'histoire des sciences."

138 MWG I/2, 304, Anm. 45 ; 315 ; GASWG 21, 144 et 272 [WEBER, Économie et société dans l'Antiquité $106 \mathrm{sv}, 250,385]$.

139 GASWG 20 [trad. in Économie et société dans l'Antiquité 105]. Il est notamment question d'esclaves enchaînés dans Columelle I, 6, 3; 1, 9, 4. Le monitor cité chez Columelle I, 9, 7 est appelé Antreiber et Treiber, "conducteur ", dans MWG I/2, 346 et GASWG $298=$ Économie et société dans l'Antiquité 71 et "fouetteur " dans WuG 686 (chapitre "Sociologie de la domination"). Le motif du fouet, qui manque chez Columelle, pourrait aussi venir de parallèles modernes rapprochés des preuves antiques de l'entravement et des prisons d'esclaves (voir à ce sujet BACKHAUS [1989]).

140 GASWG 298. [trad. in Économie et société dans l'Antiquité 71].

141 WuG 85 ; cf. 524. [WeBER, Économie et société, coll. Agora, vol. 1, 212 sv.]. 
régulièrement approvisionné et de très grands domaines agricoles du type plantations, comme à Carthage 142, à Rome et, pour une part, dans les colonies européennes modernes et en Amérique du Nord. "Le tarissement du marché des esclaves (comme conséquence de la pacification de l'Empire) a mené au rétrécissement des plantations antiques ; en Amérique du Nord, les mêmes circonstances ont conduit à une chasse constante aux territoires à bon marché [... 143."

Ce parallèle revient à un autre endroit du même ensemble de textes ${ }^{144}$ : « [...] l'esclavagisme capitaliste de l'Antiquité disparaît avec la pacification de l'Empire [...] ; l'esclavage capitaliste des États sudistes est condamné à disparaître lorsque les terres cultivables se raréfièrent et que la fin de l'importation d'esclaves entraîna une augmentation monopolistique des prix ${ }^{145}$. " La guerre civile américaine n'a sur ce point fait qu'anticiper une nécessité économique qui avait déjà été induite par l'arrêt des importations d'esclaves en 1808. Si, dans son texte de 1909, Weber avait encore signalé en passant que dans les États du Sud, l'" élevage des esclaves a été pratiqué de façon massive à des fins spéculatives 146 ", l'importance de ce facteur était à présent ignorée.

L'abolition de l'esclavage à l'époque moderne aurait été déterminée par des facteurs économiques :

« La suppression de l'esclavage [...] est avant tout le produit du déplacement du centre de gravité de la domination économique mondiale dans des domaines où le travail servile n'est pas rentable en raison du coût élevé de l'entretien de l'esclave ; d'un autre côté, le système du travail salarié avec sa menace de licenciement et de chômage entraîne une

142 L'hypothèse de Weber selon laquelle Carthage avait inventé la plantation gérée au moyen d'esclaves " disciplinés militairement " et "soumis à une usure sans scrupule ", et que ce modèle avait été repris par les Romains (GASWG 238 sv. [WEBER, Économie et société dans l'Antiquité $348 \mathrm{sv}$.]) remonte certainement à Mommsen ; cf. Th. MOMMSEN (1902) I, 490 et 499 sv. ; II, 80. La question de savoir si les rares sources - cf. WeSTERMANN (1935) 945 sv. - suffisent à prouver cette image de l'agriculture carthaginoise reste ouverte.

143 WuG 95 [trad. in Économie et société I, 231 (trad. mod.)].

144 Seule la " $1^{\mathrm{e}}$ partie " d'Économie et société a été publiée par Weber lui-même en 1919/20 ; la « $2^{\mathrm{e}}$ partie » se compose de textes inédits de Weber, écrits entre 1910 et 1914 environ; cf. W. J. Mommsen (2000). On ne constate pas, concernant les propos sur l'esclavage, de différence entre les strates successives de l'œuvre.

145 WuG 415 [trad. in WeBER, Sociologie du droit 73]. - Cette thèse selon laquelle l'emploi d'esclaves non qualifiés impliquait une exploitation abusive du sol, qui devait ensuite être compensée par la mise en exploitation de nouveaux territoires, était également un topos du débat sur l'abolition - voir par exemple CAIRNES (1863/2003) 54 sv. - elle apparaît d'ailleurs aussi chez Marx et Engels (MEW 15, 335 sv. [MARX, La Guerre civile nordaméricaine] ; 20, 164 [ENGELS, Anti-Dühring]). Le phénomène ne s'explique cependant pas par une particularité du travail servile, mais plutôt par la relation entre le prix élevé des esclaves et le prix peu élevé des terrains ; cf. BACKHAUS (1974) 139 sv. Quant à savoir si c'est une explication suffisante, nous ne trancherons pas. GASWG 19 [trad. in Économie et société dans l'Antiquité 105]. 
contrainte indirecte de travailler; de ce fait la contrainte directe est considérée comme moins efficace pour extorquer un travail de qualité de l'assujetti sans courir les grands risques des investissements nécessaires pour le travail servile ${ }^{147}$."

Dans plusieurs passages du même ouvrage, Weber trace aussi - à nouveau en accord avec Marx et avec l'argumentation abolitionniste, mais avec une mise en exergue plus forte du phénomène antique - un parallèle entre la situation romaine et celle des États sudistes américains en ce qui concerne l'apparition, suite à l'augmentation de l'esclavage, d'un sous-prolétariat d'hommes libres déclassés. Celui-ci ne présente aucune propension pour l'activité productive, mais il manifeste un intérêt particulier pour le maintien des frontières de leur statut par rapport aux esclaves ; dans l'Antiquité, cet intérêt se concrétise par la participation aux distributions de céréales et aux jeux ; en Amérique du Nord, en entretenant un sentiment de supériorité raciste. Le concept de " poor white trash 148 ", ici utilisé par Weber pour désigner le sous-prolétariat américain, provient, comme nous l'avons dit, de la littérature abolitionniste, dans laquelle les effets destructeurs de l'esclavage sur la société sont déplorés.

La mise en parallèle par Weber de l'esclavage antique et américain apparaît de nouveau clairement dans son Histoire économique. Il ne s'agit cependant pas d'un texte original de Weber, mais de notes d'auditeurs d'un cours donné durant l'hiver 1919/20 à Munich ${ }^{149}$. Un passage sur la plantation comme forme capitaliste de propriété foncière traite de l'esclavage carthagino-romain et nord-américain en tant que types classiques de plantations à part entière :

"La difficulté majeure de l'exploitation réside dans le recrutement des travailleurs. Ceux-ci n'ont pas de famille et ne se reproduisent donc pas eux-mêmes. L'existence d'une telle plantation est par conséquent fonction de la possibilité d'une chasse à l'esclave, soit en profitant d'une situation de guerre, soit par l'exploitation périodique d'un grand territoire où effectuer une telle chasse, comme ce fut le cas de l'Afrique avec la traite des Noirs. [...] L'esclavage n'est rentable que s'il est pratiqué avec la plus stricte discipline et s'il est associé à une exploitation sans scrupules ; il présuppose, de surcroît, la possibilité à la fois d'approvisionner et de nourrir les esclaves au moindre coût et de pratiquer largement une culture extensive (Raubbau), ce qui, à son tour, suppose qu'il n'y ait pas de limites au terroir dont on peut disposer ${ }^{150}$."

147 WuG 415 [trad. in WeBER, Sociologie du droit 72 (trad. mod.)].

148 WuG 178 [WeBER, Économie et société, coll. Agora, vol. 1, 393] ; MWG I/22-1, 178 ; MWG I/22-5, 254 ; cf. Wg 85 [trad. in WeBER, Histoire économique 110].

149 Le texte contient dans l'ensemble de nombreux passages et affirmations apparaissant ailleurs dans les écrits de Weber. En revanche, les références bibliographiques qui y sont insérées sont dues aux éditeurs.

150 Wg 82 sv. et 85 [trad. in Weber, Histoire économique 106, 107 et 110]. 
La légalisation du mariage des esclaves dans l'Antiquité d'une part, l'interdiction de leur importation aux États-Unis de l'autre ont nécessairement conditionné la fin de l'esclavage 151 . Concernant les conditions modernes, la théorie du sabotage apparaitt également dans ce passage : "culture nègre " signifiait " exploitation abusive du sol (Raubbau) car le nègre, à qui on ne pouvait confier aucun instrument moderne, ne travaillait la terre quavec des instruments primitifs 152 ".

Weber s'aventure bien plus imprudemment que Marx dans la comparaison entre esclavage romain et américain ; comme Marx avant lui, il s'appuie, concernant les conditions américaines, sur des conceptions qui peuvent aujourd'hui être considérées comme réfutées. L'esclavage dans les États sudistes n'était pas devenu économiquement obsolète du fait qu'il s'était avéré non rentable pour les propriétaires d'esclaves. Le fait que le Sud, avec son économie agraire, se soit laissé progressivement distancier dans le développement économique par le Nord industriel représente une tout autre question. L'hypothèse selon laquelle l'économie esclavagiste nord-américaine avait été condamnée à disparaître avec la fin des importations d'esclaves n'est pas soutenable telle quelle. Elle ne tient pas compte des deux facteurs suivants : d'une part, on payait déjà très cher pour les esclaves africains au XVIII siècle ${ }^{153}$, d'autre part, la proportion de femmes était élevée parmi les esclaves des États sudistes américains, puisque celles-ci étaient, de la même façon, enrôlées dans les champs (en tout cas dans les plantations de coton ${ }^{154}$ ) et que l'on autorisait la formation de familles avec, à la clé, un degré élevé de reproduction.

Ce que les opposants et les partisans de cette " peculiar institution " espéraient ou craignaient respectivement, pendant la première moitié du XIXe siècle, c'était certainement qu'une interdiction de l'importation élimine l'esclavage à plus long terme dans l'ensemble des États-Unis ${ }^{155}$. Mais au cours des vingt années qui séparèrent l'adoption de la constitution fédérale et la proclamation de l'interdiction, le flux d'esclaves africains atteignit un sommet, ce qui entraîna, dans un second temps, une augmentation remarquable de la reproduction naturelle, encouragée à dessein par les propriétaires eux-mêmes. Le nombre d'esclaves passa d'environ 700000 en 1790 à 1,2 million en 1810 et à environ 4 millions en 1860 - et cela malgré l'abolition de l'esclavage dans les États nordistes ${ }^{156}$. Non seulement ce fait avait été, comme nous nous l'avons vu plus

$151 \mathrm{Wg} 85 \mathrm{sv}$. [trad. in WEBER, Histoire économique 110].

152 Wg 85. [trad. in Histoire économique 109 sv. (trad. mod.)]. Voir aussi WuG 95 [Économie et société I, 230] : faute de responsabilité et de motivations personnelles, le travail servile était difficilement concevable avec des outils exigeants.

153 KLEIN (1990).

154 TADMAN (2000).

155 Ainsi aussi, rétrospectivement, Daniel Webster dans un discours au Sénat américain de 1850 ; cité par RAHE (1992) 638.

156 Kolchin (2003) 93. - Ainsi que le montre la comparaison avec les économies esclavagistes des Caraïbes et d'Amérique du Sud, en particulier dans les plantations de canne à sucre, les États sudistes représentent à cet égard une exception tout à fait remarquable ; TADMAN (2000). 
haut, connu de Marx, mais il était de notoriété publique à l'époque de Weber 157. Pourquoi donc ce dernier n'en a-t-il pas tenu compte dans son argumentation ?

On peut soupçonner à ce sujet que, concernant la question démographique, Weber avait élevé les anciennes observations, telles qu'il les comprenait, au rang d'état de fait universel (et qu'il les utilisait dès lors de manière axiomatique), alors que, concernant la productivité générale plus réduite de l'esclavage par rapport au travail libre, il partait de la littérature abolitionniste du XIXe siècle. En ce qui concerne ce second point, le fait que Weber se soit appuyé sur la littérature contemporaine, dans laquelle les hypothèses de fond de l'abolitionnisme étaient transposées dans l'Antiquité, constitue un indice significatif. Cela vaut en particulier pour les travaux de deux savants italiens étiquetés comme marxistes, l'antiquisant Ettore Ciccotti 158 et l'économiste Achille Loria ${ }^{159}$, qui avaient abondamment lu non seulement Marx, mais aussi la littérature utilisée par celui-ci, en particulier John Elliott Cairnes 160 .

Nous ne pouvons certes reprocher à Weber de n’avoir pas connu les résultats des nouvelles recherches sur l'économie esclavagiste menées à partir des années 1960. Nous ne pouvons pas davantage lui objecter que la recherche sur l'histoire ancienne est parvenue (y compris en ce qui concerne les différentes régions de l'Empire) à une image bien plus nuancée du recours à la main-d'œuvre dans l'agriculture romaine, qui tient compte de l'emploi simultané d'esclaves, avec l'intervention de fermiers et de journaliers, tant dans la république tardive que sous l'Empire, de sorte qu'il n'est plus question d'un remplacement général des esclaves par des colons 161.

157 SARTORIUS VON WALTERSHAUSEN (1910) 907. L'article trahit une tendance au racisme ; celle-ci est encore plus claire dans l'article de Grünberg inséré dans le même ouvrage, qui s'irrite de ce qu'après la guerre civile américaine, " quatre millions de nègres pour la plupart presque abrutis » aient été faits citoyens ; GRÜNBERG (1911), 540.

158 CiCCOTTI (1899). En 1898, Ciccotti (1863-1939) perdit sa chaire milanaise à cause de ses engagements socialistes. En 1901, après avoir manqué d'autres postes pour des raisons politiques, il devint professeur à Messine ; voir RICCARDI (1997) 207 sv.

159 LORIA (1896). - Loria (1857-1943), qui avait rencontré Marx et Engels à Londres mais n'était guère apprécié d'eux en retour, fut, en raison de son article sur Marx de 1883, traité à diverses reprises par Engels de faussaire et en même temps de plagiaire des idées de Marx ; MEW 25, 25 [MARX, Le Capital] ; 36, 19. On trouve en outre de nombreuses remarques péjoratives sur Loria dans les lettres d'Engels, notamment dans MEW 39, 325 et 38,57 , où il est respectivement traité de " charlatan " et de "grande gueule ".

160 Sur la connaissance par Weber de ces travaux : GASWG 279 [Économie et société dans l'Antiquité 391 sv.]. Ibid. à propos du livre de Ciccotti qu'il qualifie d'" application au monde antique des points de vue de Slave Power de Cairnes dont la valeur est indéniable, en dépit d'un certain manque de précision et de sel ", [trad. in Économie et société dans l'Antiquité 392 (trad. mod.)]. Sur l'influence de Cairnes sur Ciccotti et Loria, voir BaCKHAus (1975) 550 sv. ; MaZZa (1979) ; RiCCARdi (1997) 236 sv.

161 Cf. l'aperçu de Pleket (1990) 99 sv. et WhitTaker/Garnsey (1998) 294 sv. Voir aussi, sur l'histoire de la recherche, MARCONE (1988). 
Il faut toutefois constater que Weber, qui a, dans ses comparaisons et dans ses typologies historiques, fortement souligné les différences entre les divers phénomènes historiques au point de les traiter souvent de manière plus détaillée que les points communs, n'a apparemment consacré aucune réflexion à la problématique d'un idéaltype universel d' "économie de plantation ». Dans ce cadre, les différences relatives aux méthodes agricoles, à l'organisation du travail et de la discipline, à la taille des exploitations, à l'emploi d'ouvriers libres ou de journaliers en plus des esclaves, à la structure démographique, aux conditions de vie générales, y compris dans les relations avec les propriétaires et dans les chances d'affranchissement auraient pu être thématisées, de même que, pour la période moderne, les grandes différences entre les situations observées dans les Caraïbes, au Brésil et aux États-Unis 162.

Quant à savoir si la référence à l'état de la recherche à la fin du XIXe siècle suffit à expliquer pourquoi, dans ce cas, Weber manie de façon si schématique les correspondances, par-delà les frontières historiques, et aplanit les différences entre les phénomènes historiques spécifiques, je ne me prononcerai pas ${ }^{163}$. Finalement, Weber était, d'après Adolf von Harnack, " au niveau international, entre 1890 et 1920, l'homme possédant la plus forte capacité de consommation scientifique et une singulière faculté de s'approprier tous les sujets $164 »$.

162 Dans les États sudistes américains, les plantations de tabac employaient en moyenne moins de vingt esclaves, celles de coton rarement plus de cinquante; les planteurs de coton habitaient sur leurs terres, à proximité immédiate de leurs esclaves, ce qui renforçait leur paternalisme. Même dans les plantations de grande taille, l'engagement de surveillants d'esclaves n'était pas la règle ; les esclaves avaient une espérance de vie relativement bonne ; FogEL/ENGERMAN (1974) 25 sv. et 200 sv. Leurs conditions se distiguent nettement de la situation des esclaves dans les Caraïbes (cf. WIRZ [1984] 137 et 144 sv.) ainsi que de celles des esclaves des latifundia romains (cf. CrOUCH [1985] 9 sv. ; HARRIs [1999]).

163 Lors de son voyage en Amérique en 1904, Weber s'est principalement intéressé à la discrimination continuelle dont souffrait la population noire ; cf. Marianne WEBER (1926) 307 sv. Il déclara par la suite (tout à fait sérieusement, en discutant les théories contemporaines de la « race ») que, selon son expérience personnelle, l'« odeur des nègres » n'était pas une réalité, mais une discrimination raciste : " une invention des États du Nord »; GASS 460 [trad. in Max Weber et les relations ethniques 179; WuG 239 = MWG I/22-1, 179 [trad. in WeBER, Économie et société, coll. Agora, vol. 2 134]. L'article de DuBois (1906) revient sur l'idée de Weber ; W. J. Mommsen (1998) 96 sv. DuBois, qui s'engagea en faveur de l'égalité sociale entre populations de couleur et blanches, avait étudié en Allemagne (il fut sans doute le premier noir américain dans ce cas). La rédaction de l'Archiv für Sozialwissenschaft und Sozialpolitik le décrivit comme "l'un des principaux représentants scientifiques des nègres américains".

164 Selon les termes de Harnack en 1920 ; cité chez Heuß (1951) 503. - Voir aussi NeumanN (1923) 162 : "Max Weber [a tracé] le réseau de routes nécessaire à ses expéditions de conquistador, grâce auxquelles l'impulsion impérialiste de ses talents élèvera, d'une manière inouïe, des connaissances disciplinaires au rang de science totale. » 
Mon intention était de montrer, par le biais des exemples de Marx et de Weber en tant qu'éminents théoriciens de l'économie du XIXe siècle et du début XXe, combien la réflexion sur l'esclavage antique a été associée au discours esclavagiste du XIXe siècle.

Une tout autre question, que je ne pouvais ni ne souhaitais traiter dans cet exposé, consiste à se demander sous quels angles de vue il serait aujourd'hui pertinent de mettre en relation les résultats des amples recherches menées ces cinquante dernières années sur l'esclavage moderne avec les phénomènes antiques 165 . Un effet positif de cette moisson réside sûrement dans le fait que l'on a pris ses distances vis-à-vis des prises de position globales sur l'efficacité ou la non-efficacité économique de l'esclavage, sur l'importance, pour l'économie esclavagiste de l'Antiquité romaine, de la reproduction naturelle ${ }^{166}$, des abandons ou des rapts d'enfants, ainsi que de la pratique selon laquelle des personnes se vendaient elles-mêmes en esclavage ${ }^{167}$ et, d'une manière plus générale, de la traite des esclaves 168 ; on a en outre introduit dans l'analyse ${ }^{169}$ la catégorie de " mort sociale 170 ". Il est vrai qu'il existe aussi un risque : celui que, de l'exemple américain en matière de taux élevés de reproduction naturelle, l'on tire des déductions hasardeuses sur l'esclavage romain ${ }^{171}$. Le recours à l'histoire des sciences sociales ne résout pas les problèmes de recherche actuels, mais il permet de mieux identifier les potentialités et les risques de la comparaison historique.

\begin{tabular}{l} 
Wilfried NiPPEL \\
\hline Institut für Geschichtswissenschaften \\
Humboldt-Universität zu Berlin \\
Unter den Linden 6 \\
D-10099 Berlin \\
NippelW@geschichte.hu-berlin.de
\end{tabular}

165 Voir en général Finley (1981a), SHAW (1998) puis WeILER (2003). - BARROW (1928), 230-236 était encore parti de la justesse de l'analyse de Cairnes, dont il avait tiré des conclusions pour l'Antiquité romaine.

166 Bradley (1987) ; Herrmann-Otto (1994). - Depuis la fin des années 1950, Moses Finley a rappelé à plusieurs reprises avec insistance que nos connaissances sur l'esclavage moderne contredisaient le mythe d'une population d'esclaves ne pouvant se reproduire de manière naturelle ; voir par exemple FINLEY (1981b) 103 sv. ; IDEM (1961).

RAMIN/VEYNE (1981)

168 HARRIS (1980).

169 En général : PATTERSON (1982); sur l'Antiquité romaine : HERRMANn-OTto (2002).

170 Pour l'emploi de la comparaison avec les Temps modernes concernant les risques de révolte d'esclaves dans la Grèce antique, cf. CARTLEDGE (1985).

171 Cette tendance apparaît notamment chez SCHEIDEL (1997) ; pour une critique, voir HARRIS (1999). 


\section{Bibliographie}

ADAmS, Willi Paul, "Das Gleichheitspostulat in der Amerikanischen Revolution”, Historische Zeitschrift 212 (1971), 59-99.

ARMitage, David, “That excellent forme of government. New lights on Locke and Carolina”, Times Literary Supplement $\mathrm{n}^{\circ}$ 5299, 22. 10. 2004, 14-15.

Audring, Gert/SperL, Richard, "Welch sonderbares Geschick diese library hat". Weitere Bände aus der Bibliothek von Karl Marx entdeckt, in MEGA Studien 1997/1, 120-128.

BaCKHaUs, Wilhelm, Marx, Engels und die Sklaverei, Düsseldorf 1974.

BACKHAUS, Wilhelm, John Elliott Cairnes und die Erforschung der antiken Sklaverei”, Historische Zeitschrift 220 (1975), 543-567.

BACKHAUS, Wilhelm, "Servi vincti", Klio 71 (1989), 321-329.

BALDwIn, Barry, "The Young Karl Marx on Roman History", Archiv für Kulturgeschichte 70 (1988), 101-107.

BarClay, John M. G., Paul, "Philemon and the Dilemma of Christian Slave-Ownership", New Testament Studies 37 (1991), 161-186.

BARROw, Reginald H., Slavery in the Roman Empire, Londres 1928.

Baumgarten, Eduard, Max Weber. Werk und Person, Tübingen 1964.

BENOt, Yves, La révolution française et la fin des colonies, Paris 1988.

BERDING, Helmut, "Die Ächtung des Sklavenhandels auf dem Wiener Kongreß 1814/15", Historische Zeitschrift 219 (1974), 265-289.

BiEZUNSKa MALOWIST, Iza, "Les recherches sur l'esclavage ancient et le mouvement abolitionniste européen", in Jan BURIAN/Ladislav VIDMAN (éd.), Antiquitas graeco-romana ac tempora nostra, Prague 1968, 161-167.

BlaCKBURn, Robin, The Overthrow of Colonial Slavery, 1776-1848, Londres 1988.

BöCKH, August, Die Staatshaushaltung der Athener, 2 vol., Berlin 1886 [1" éd. 1817].

BRADLEY, Keith R., "On the Roman Slave Supply and Slavebreeding”, in Moses I. Finley (éd.), Classical Slavery (Slavery and Abolition, Special Issue 8), Londres 1987, 42-64.

BRUHNS, Hinnerk (1998), “À propos de l'histoire ancienne et de l'économie politique chez Max Weber”, in Max WeBER, Économie et société dans l'antiquité, Paris 1998, 9-59.

BÜCHER, Karl, "Zur griechischen Wirtschaftsgeschichte" [1901], in IDEM, Beiträge zur Wirtschaftsgeschichte, Tübingen 1922, 1-97.

Cambiano, Giuseppe, "Aristotle and the Anonymous Opponents of Slavery", in Moses I. FINLEY (éd.), Classical Slavery (Slavery and Abolition, Special Issue 8), Londres 1987, 2241.

CAIRNES, John Elliott, Slave Power. Its character, career and probable designs, Columbia, SC, 2003 [réimp. de la 2e éd., 1863].

Cartledge, Paul, "Rebels \& Sambos in Classical Greece. A comparative view", in Crux. Essays presented to G. E. M. de Ste. Croix on his 75th birthday, Exeter 1985, 16-46.

CicCOTTI, Ettore, Il tramonto della schiavitù nel mondo antico, Turin 1899.

Comte, Auguste, Die Soziologie. Die positive Philosophie im Auszug. éd. Friedrich Blaschke, Leipzig 1933.

[ComTe, Auguste, La Sociologie, Paris, Félix Alcan, 1897]. 
Crouch, Barry A., “'Booty Capitalis' and Capitalism's Booty. Slaves and slavery in Ancient Rome and the American South", Slavery \& Abolition 6 (1985), 3-24.

Davis, David Brion, The Problem of Slavery in Western Culture, Ithaca, N. Y. 1966.

DAVIS, David Brion, "Slavery and the Post-World War II Historians", Daedalus 103 (1974), 116.

DavIS, David Brion, The Problem of Slavery in the Age of Revolution (1770-1823), Ithaca, N. Y. 1975.

DAVIS, David Brion, Challenging the Boundaries of Slavery, Cambridge, Mass. 2003.

DEIßLER, Johannes, Antike Sklaverei und deutsche Aufklärung im Spiegel von Johann Friedrich Reitemeiers "Geschichte und Zustand der Sklaverey und Leibeigenschaft in Griechenland"1789 (Forschungen zur antiken Sklaverei 33), Stuttgart 2000.

Deißler, Johannes, "Friedrich Nietzsche und die antike Sklaverei", in Heinz BelleN/Heinz HeINEN (éd.), Fünfzig Jahre Forschungen zur antiken Sklaverei an der Mainzer Akademie 1950-2000 (Forschungen zur antiken Sklaverei 35), Stuttgart 2001, 457-485.

Den Hollander, A. N. J., "Countries Far Away - Cognition at a Distance", Comparative Studies in Society and History 9 (1967), 352-376.

DigGins, John Patrick, "Slavery, Race and Equality. Jefferson and the pathos of the enlightenment", American Quarterly 28 (1976), 206-228.

DresCher, Seymour, "Trends in der Historiographie des Abolitionismus", Geschichte und Gesellschaft 16 (1990), 187-211.

DresCHER, Seymour, "Whose Abolition ? Popular pressure and the ending of the British slave trade”, Past \& Present 143 (1994), 136-166.

DresCHER, Seymour, The Mighty Experience. Free labor versus slavery in British emancipation, Oxford 2002.

DuBois, W. E. B., "Die Negerfrage in den Vereinigten Staaten", Archiv für Sozialwissenschaft und Sozialpolitik 22 (1906), 31-79.

Dumont, Jean-Christian, "Esclavage blanc, esclavage noir", Bulletin de l'Association Guillaume Budé 1988/II, 174-194.

DumOnT, Jean-Christian, "La Révolution française et Rome", Studi Italiani di Filologia Classica $85=3$. ser., 10 (1992), 487-512.

ERdmann, Karl Dietrich, Die Ökumene der Historiker. Geschichte der internationalen Historikerkongresse und des Comité International des Sciences Historiques (Abhandlungen der Akademie der Wissenschaften Göttingen, Phil.-Hist. Kl. III, 158), Göttingen 1987.

Finkelman, Paul, Slavery and the Founders. Race and liberty in the age of Jefferson, Armonk, N. Y. 1996.

Finley, Moses I., "The Signifance of Ancient Slavery", Acta Antiqua Academiae Scientiarum Hungaricae 9 (1961), 285-286.

FInley, Moses I., Die Sklaverei in der Antike, Munich 1981 (= FInLEY 1981a). [Ancient Slavery and Modern Ideology, 1980 ; rééd. de Brent Shaw, Princeton, N. J. 1998].

FinleY, Moses I., "Was Greek Civilization Based on Slave Labour ?”, in IDEM., Economy and Society in Ancient Greece, Londres 1981, 97-115 (= FINLEY 1981b). [1e éd. : Historia 8 (1959)].

Fitzhugh, George, Cannibals All! Or : Slaves without masters, éd. par C. Vann Woodward, Cambridge, Mass. 1988 [1éd. 1857]. 
Fogel, Robert W./Engerman, Stanley L., Time on the Cross. The economics of American negro slavery, Londres 1974.

Fogel, Robert W., The Slavery Debates, 1952-1990. A retrospective, Baton Rouge 2003.

Fox-Genovese, Elizabeth/Genovese, Eugene D., "The Divine Sanction of Social Order : Religious Foundations of the southern slaveholders' world view", Journal of the American Academy of Religion 55 (1987), 211-233.

Garnsey, Peter D. A., Ideas of Slavery from Aristotle to Augustine, Cambridge 1996.

GARS, cf. WEBER, Max.

GASS, cf. WEBER, Max.

GASWG, cf. WEBER, Max.

GAWL, cf. WEBER, Max.

Geggus, David, "Racial Equality, Slavery, and Colonial Secession during the Constituent Assembly", American Historical Review 94 (1989), 1290-1308.

GerTeIS, Louis S., Morality and Utility in American Anti-Slavery Reform, Chapel Hill, N. C. 1987.

Goldenberg, David M., The Curse of Ham. Race and slavery in early Judaism, Christianity, and Islam, Princeton, N. J. 2003.

GPS, cf. Weber, Max.

GreENE, John C., "The American Debate on the Negro's Place in Nature, 1780-1815”, Journal of the History of Ideas 15 (1954), 384-396.

Grotius, Hugo, De Jure belli ac pacis libri tres 1625. Drei Bücher vom Recht des Krieges und des Friedens, hrsg. v. Walter Schätzel (Klassiker des Völkerrechts in modernen deutschen Übersetzungen 1), Tübingen 1950.

GRÜNBERG, Carl, "Sklaverei”, in Handwörterbuch der Staatswissenschaften, 7 (1911), 524-541.

Harrington, J. Drew, "Classical Antiquity and the Proslavery Argument", Slavery \& Abolition 10 (1989), 60-72.

Harris, William V., "Towards a Study of the Roman Slave Trade", Memoirs of the American Academy in Rome 36 (1980), 117-140.

HARrIS, William V., "Demography, Geography and the Sources of Roman Slaves", Journal of Roman Studies 89 (1999), 62-75.

Hartfield, Marianne, "New Thoughts on the Proslavery Natural Law Theory. The importance of history and the study of ancient slavery", Southern Studies 22 (1983), 244-259.

HAYNES, Stephen R., Noah's Curse. The biblical justification of American slavery, Oxford 2002.

HeInen, Heinz, "Das Ende der Alten Welt im Rahmen der Gesamtentwicklung der sowjetischen Althistorie", in IDEM (éd.), Die Geschichte des Altertums im Spiegel der sowjetischen Forschung (Erträge der Forschung 146), Darmstadt 1980, 256-340.

HenNis, Wilhelm, La Problématique de Max Weber. Traduit par Lilyane Deroche-Gurcel, Paris 1996 [original allemand 1987].

HERRMANN-OTTO, Elisabeth, Ex ancilla natus. Untersuchungen zu den "hausgeborenen“ Sklaven und Sklavinnen im Westen des römischen Kaiserreiches (Forschungen zur antiken Sklaverei 24), Stuttgart 1994.

Herrmann-Otto, Elisabeth, "Der soziale Tod : Leben am Rande der römischen Gesellschaft", Orbis Iuris Romani 7 (2002), 20-41.

Heuß, Theodor, Weber, Max, in ID., Deutsche Gestalten. Studien zum 19. Jahrhundert, Tübingen 1951, 501-509. 
Honigsheim, Paul, "Max Weber in Heidelberg”, in René KÖNIG/Johannes WinCKeLMANN (éd.), Max Weber zum Gedächtnis. Materialien und Dokumente zur Bewertung von Werk und Persönlichkeit, Cologne 1963, 161-271.

Hume, David, "Of the Populousness of Ancient Nations [1752]", in David Hume, The Philosophical Works, éd. par Thomas H. Green/Thomas H. Rose, Bd. III, Londres 1882 [ND Aalen 1964], 381-463.

Hunting, Claudine, “'The Philosophes' and Black Slavery 1748-1765”, Journal of the History of Ideas 39 (1978), 405-418.

Huston, James L., "Abolitionists, Political Economists, and Capitalism”, Journal of the Early Republic 20 (2000), 487-521.

JefFerson, Thomas, Writings, éd. par Merrill D. Peterson, New York 1984.

Jordan, Winthrop D., White over Black. American attitudes toward the negro, 1550-1812, Chapel Hill, N. C. 1968.

KaUTSKY, Karl, Der Ursprung des Christentums. Eine historische Untersuchung, Stuttgart 1908.

KaUTSKY, Karl, "Sklaverei und Kapitalimus", Die Neue Zeit, 29, 2 (1911) 713-725 [Recension, sous forme d'essai, de la traduction allemande de Ciccotti].

KLEIN, Herbert S., "Neuere Interpretationen des atlantischen Sklavenhandels", Geschichte und Gesellschaft 16 (1990), 141-160.

KolChIN, Peter, "Die südstaatliche Sklaverei vor dem amerikanischen Bürgerkrieg und die Historiker. Zur Debatte 1959-1988", Geschichte und Gesellschaft 16 (1990), 161-186.

KolChIn, Peter, American Slavery, 1619-1877, New York 2003.

LENCMAN, Jakov A., Die Sklaverei im mykenischen und homerischen Griechenland (Übersetzungen ausländischer Arbeiten zur antiken Sklaverei 1), Wiesbaden 1966.

LenIN, Wladimir Iljitsch, "Über den Staat”, in Lenin, Werke, 29 : März-August 1919, éd. par l'Institut für Marxismus-Leninismus beim ZK der KPdSU, Berlin 1961, 460-484.

LEVINE, Norman, "The German Historical School of Law and the Origins of Historical Materialism", Journal of the History of Ideas 48 (1987), 431-451.

LOCKE, John, Two Treatises of Government, éd. par Peter Laslett, Cambridge 1988.

LORIA, Achille, "Die Sclavenwirthschaft im modernen Amerika und im europäischen Alterthume", Zeitschrift für Social- und Wirtschaftsgeschichte 4 (1896), 67-118.

McKeE Evans, William, "From the Land of Canaan to the Land of Guinea. The strange odyssee of the "Sons of Ham"”, American Historical Review 85 (1980), 15-43.

MarCone, Arnaldo, Il colonato tardoantico nella storiografia moderna da Fustel de Coulanges ai nostri giorni (Biblioteca di Athenaeum 7), Côme 1988.

Marra, Realino, Capitalismo e anticapitalismo in Max Weber. Storia di Roma e sociologia del diritto nella genesi dell'opera weberiana, Bologne 2002.

MarX, Karl/Engels, Friedrich, Werke, éd. par le Zentralinstitut für Marxismus-Leninismus beim ZK der SED, Berlin 1956 sv. (= MEW).

MarX, Karl/EngELS, Friedrich, Gesamtausgabe, nouvelle édition Berlin 1975 sv. (= MEGA).

[ENGELs, Friedrich, Anti-Dühring, M. E. Dühring bouleverse la science, Paris 1950 (CEuvres complètes de Friedrich Engels, trad. d'Émile Bottigelli)].

[MarX, Karl, Le 18 Brumaire de Louis Bonaparte, Paris 1956 [pas de mention de traducteur]].

[MARX, Karl, Le Capital, XIV, Paris 1946 (trad. Molitor)]. 
[Marx, Karl, Euvres, I. Économie (Préf. par François Perroux ; éd. établie par Maximilien Rubel) et II. Économie (Éd. établie et annotée par Maximilien Rubel), Paris 1965 et 1968 (Bibliothèque de la Pléiade 164 et 204)].

[MarX, Karl/Engels, Friedrich, Correspondance (publ. sous la responsabilité de Gilbert Badia et Jean Mortier), vol. 4 (juillet 1853 - juin 1857), Paris 1978 et vol. 6 (1860-1861), Paris 1978].

[MARX, Karl/Engels, Friedrich, L'Idéologie allemande. Thèse sur Feuerbach (intr. de Jacques Milhau, trad. de Gilbert Badia), Paris 1982].

[MarX, Karl, Lassalle, Fernand, Correspondance K. Marx-F. Lassalle, 1848-1864, Paris 1977 (Prés., trad. et annoté par Sonia Dayan-Herzbrun)].

Mason, Matthew, "The Battle of the Slaveholding Liberators. Great Britain, the United States and Slavery", William and Mary Quarterly 3e série., 59 (2002), 665-697.

MAZZA, Mario, “Tra diritto e storia. 'Il tramonto della schiavitù nel mondo antico' di Ettore Ciccotti”, Klio 61 (1979), 57-83.

MAZZA, Mario, "Meyer vs. Bücher : Il dibattito sull'economia antica nella storiografia tedesca tra otto e novecento", Società e Storia 29 (1985), 508-546.

MEGA, cf. MARX, Karl/ENGELS, Friedrich.

MEINERS, Christoph, Über die Natur der afrikanischen Neger, und die davon abhängende Befreyung oder Einschränkung der Schwarzen, éd. par. Frank Schäfer, Hanovre 1997 [Réimpr. de : Göttingisches Historisches Magazin 6, 1790].

Menze, Clemens, "Wilhelm von Humboldt und die antike Sklaverei. Eine Stellungnahme zu einer Diskussion über die Rezeption des griechischen Altertums im deutschen Humanismus", Vierteljahrsschrift für wissenschaftliche Pädagogik 63 (1987), 319-339.

MEW, cf. MARX, Karl/EnGELs, Friedrich.

MeYer, Eduard, "Die Sklaverei im Altertum", in IDEM., Kleine Schriften I, Halle 1924, 169-212 [1 éd. 1898].

MiLl, John Stuart, Principles of Political Economy, Amherst, N. Y. 2004 [réimpr. de l'édition de New York 1900].

Millar, John, The Origin of the Distinction of Ranks, Édimbourg 1806 [réimpr. Aalen 1986].

Miller, William L., "J. E. Cairnes on the Economics of American Negro Slavery", Southern Economic Journal 30 (1963/64), 333-341.

MoHL, Robert [von], "Die Aufhebung der Sklaverei in den englischen und französischen Kolonieen", Zeitschrift für die gesammte Staatswissenschaft 1 (1844), 478-518.

Mommsen, Theodor, Römische Geschichte, Berlin 1902 [réimpr. Munich 1976].

[Mommsen, Theodor, Histoire romaine. I. Des commencements de Rome jusqu'aux guerres civiles, Paris, Robert Laffont, 1985 (éd. présentée et établie par Claude Nicolet)].

[MommSEn, Theodor, Histoire romaine. II. La monarchie militaire, Paris, Robert Laffont, 1985 (éd. présentée et établie par Claude Nicolet)].

Mommsen, Wolfgang J., "Max Weber und die Vereinigten Staaten von Amerika”, in Ragnhild FIEBIG-VON HASE/Jürgen HeIDEKING (éd.), Zwei Wege in die Moderne : Aspekte der deutschamerikanischen Beziehungen 1900-1918, Trèves 1998, 91-103.

Mommsen, Wolfgang J., "Max Weber's "Grand Sociology" : The origins and composition of "Wirtschaft und Gesellschaft. Soziologie"”, History \& Theory 39 (2000), 364-383.

Morgan, Edmund S., "Slavery and Freedom. The American paradox", Journal of American History 59 (1972/73), 5-29. 
Morgan, Kenneth, "Slavery and the Debate over Ratification of the United States Constitution”, Slavery \& Abolition 22 (2001), 40-65.

MWG =Max Weber Gesamtausgabe ; WeBER, Max.

Neumann, Carl, "Zum Tode von Ernst Troeltsch", Deutsche Vierteljahrsschrift für Literaturwissenschaft und Geistesgeschichte 1 (1923), 161-171.

Nieman, Donald G., Promises to Keep. African Americans and the constitutional order, 1776 to the present, New York 1991.

NippeL, Wilfried, Griechen, Barbaren und "Wilde". Alte Geschichte und Sozialanthropologie, Francfort 1990 (= NIPPEL 1990a).

NIPPEL, Wilfried, "Methodenentwicklung und Zeitbezüge im althistorischen Werk Max Webers", Geschichte und Gesellschaft 16 (1990), 355-374 (= NiPPEL 1990b).

NippeL, Wilfried, "Max Weber, Eduard Meyer und die "Kulturgeschichte ", in Manfred Hettling u. a. (éd.), Was ist Gesellschaftsgeschichte?, Munich 1991, 323-330.

Nippel, Wilfried, "Aristoteles und die Indios", in Christof Dipper/Martin VogT (éd.), Ringvorlesung : Entdeckungen und frühe Kolonisation (Technische Hochschule Darmstadt. Schriftenreihe Wissenschaft und Technik 63), Darmstadt 1993, 69-90.

NipPEL, Wilfried, "Max Weber, "Nationalökonom und Politiker »", in Geschichte und Gesellschaft 20 (1994), 274-298.

NipPEL, Wilfried, "Eduard Meyer, Max Weber e le origini dello stato", in Beatrice DE GERLONI (éd.), Problemi e metodi della storiografia tedesca contemporanea, Turin 1996, 175-193.

Nippel, Wilfried, "Charisma und Herrschaft", in : IDEM (éd.), Virtuosen der Macht. Herrschaft und Charisma von Perikles bis Mao, Munich 2000, 7-22, 281-289 (= NipPEL 2000a).

Nippel, Wilfried, "From Agrarian History to Cross-Cultural Comparisons. Weber on GrecoRoman Antiquity", in Stephen Turner (éd.), The Cambridge Companion to Weber, Cambridge 2000, 240-255 (= NIPPEL 2000b).

Nippel, Wilfried, "Barbaren und Indianer. Antike Ethnographie und neuzeitliches Völkerrecht”, in Tonio HÖlSCHER (éd.), Gegenwelten zu den Kulturen Griechenlands und Roms in der Antike, Munich, Leipzig 2000, 111-127 (= NIPPEL 2000c).

NipPEL, Wilfried, "Wiedergelesen : Welskopfs Produktionsverhältnisse im Alten Orient und in der griechisch-römischen Antike”, in Isolde STARK (éd.), Elisabeth Charlotte Welskopf und die Alte Geschichte in der DDR, Stuttgart 2005, 170-183.

OlmSted, Frederick Law, A Journey in the Seaboard Slave States, with Remarks on their Economy, New York 1856.

Olmsted, Frederick Law, The Cotton Kingdom. A traveller's observation on cotton and slavery in the American slave states, éd. par Arthur M. Schlesinger, Cambridge, Mass. 1996 [réimpr. de l'édition de 1861].

Osterhammel, Jürgen, Sklaverei und die Zivilisation des Westens (Carl Friedrich von Siemens Stiftung. Themen 70), Munich 2000.

Patterson, Orlando, "Slavery", Annual Review of Sociology 3 (1977), 407-449.

Patterson, Orlando, Slavery and Social Death, Cambridge, Mass. 1982.

PATTERSON, Orlando, "Freiheit, Sklaverei und die moderne Konstruktion der Rechte", in Olwen Hufton (éd.), Menschenrechte in der Geschichte, Francfort 1998, 140-193.

PleKET, Harry W., Wirtschaft, in : Friedrich ViTTINGHOFF (éd.), Europäische Wirtschafts- und Sozialgeschichte in der römischen Kaiserzeit (Handbuch der europäischen Wirtschafts- und Sozialgeschichte 1), Stuttgart 1990, 25-118. 
Rahe, Paul A., Republics, Ancient and Modern. Classical Republicanism and the American Revolution, Chapel Hill, N. C. 1992.

Ramin, Jacques/Veyne, Paul, "Droit romain et société : Les hommes libres qui passent pour esclaves et l'esclavage volontaire", Historia 30 (1981), 472-497.

RapHAEl, Lutz, "Freiheit und Wohlstand der Nationen. Alexander von Humboldts Analysen der politischen Zustände Amerikas und das politische Denken seiner Zeit", in : Historische Zeitschrift 260 (1995), 749-776.

RASKOLNIKOFF, Mouza, "La 'rivoluzione romana' e gli storici sovietici”, in EADEM, Des anciens et des modernes, éd. par Ségolène Demougin, Paris 1990, 81-94.

Resnick, Daniel P., "La Société des Amis des Noirs and the Abolition of Slavery", French Historical Studies 8 (1972), 558-569.

RICCARDI, Silvia, Die Erforschung der antiken Sklaverei in Italien vom Risorgimento bis Ettore Ciccotti (Forschungen zur antiken Sklaverei 27), Stuttgart 1997.

Rubinsohn, Wolfgang Zeev, Die großen Sklavenaufstände der Antike. 500 Jahre Forschung, Darmstadt 1993.

Russell, Frederick H., The Just War in the Middle Ages, Cambridge 1975.

Ste. Croix, Geoffrey E. M. de, The Class Struggle in the Ancient Greek World. From the archaic age to the Arab conquests, Londres 1981.

SARTORIUS VON WaLtershausen, August, "Negerfrage", in Handwörterbuch der Staatswissenschaften 6 (1910), 902-913.

SCHEIDEL, WALter, "Quantifying the Sources of Slaves in the Early Roman Empire", Journal of Roman Studies 87 (1997), 156-169.

SCHNEIDER, Helmuth, "Die Bücher-Meyer Kontroverse", in William M. CALDER/Alexander DEMANDT (éd.), Eduard Meyer. Leben und Leistung eines Universalhistorikers, Leiden 1990, 417-445.

SCHNEIDER, Helmuth, "Bücher-Meyer-Kontroverse", in Der Neue Pauly 13 (1999), 551-556.

SCHMOLLER, Gustav, Grundriß der allgemeinen Volkswirtschaftslehre, 2 vol., Munich 1923

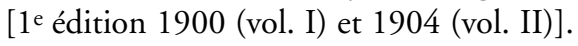

[Schmoller, Gustav, Principes d'économie politique, 5 vols. trad. par G. Platon et Leon Polack. Paris 1905-1908 (Bibliothèque internationale d'économie politique)].

SHAW, Brent D.," "A Wolf by the Ears ». M. I. Finley’s "Ancient Slavery and Modern Ideology " in historical context”, in Moses I. FINLEY, Ancient Slavery and Modern Ideology, éd. par Brent D. SHAW, Princeton, N. J., 1998, 3-74.

SMITH, Adam, An Inquiry into the Nature and Causes of the Wealth of Nations [1776]; éd. par R. H. CAMPBELl / A.S. SKINNER, Indianapolis 1981.

[SMith, Adam, Enquête sur la nature et les causes de la richesse des nations, Paris 1996.]

SMITH, Mark M., "Introduction", in CAIRNES (1863/2003), XVII-LVI.

SpaHn, Hannah, Thomas Jefferson und die Sklaverei. Verrat an der Aufklärung? (Berliner Beiträge zur Amerikanistik 12), Berlin 2002.

STAERMAN, Elena M., Die Blütezeit der Sklavenwirtschaft in der römischen Republik (Übersetzungen ausländischer Arbeiten zur antiken Sklaverei 2), Wiesbaden 1969.

STOURZH, Gerald, "Zur Konstitutionalisierung der Individualrechte in der Amerikanischen und Französischen Revolution”, in IDEM., Wege zur Grundrechtsdemokratie. Studien zur Begriffsund Institutionengeschichte des liberalen Verfassungsstaates, Vienne 1989, 155-174. 
TAdman, Michael, "The Demographic Cost of Sugar. Debates on slave societies and natural increase in the Americas", American Historical Review 105 (2000), 1534-1575.

Temperley, Howard, "Jefferson and Slavery. A study in moral complexity", in Gary L. McDowell/Sharon L. Noble (éd.), Reason and Republicanism. Thomas Jefferson's legacy of liberty, Lanham, Md. 1997, 85-99.

VitTinghofF, Friedrich, "Die Theorie des historischen Materialismus über den antiken "Sklavenhalterstaat». Probleme der Alten Geschichte bei den "Klassikern" des Marxismus und in der modernen sowjetischen Forschung", Saeculum 11 (1960), 89-131.

ViTtinghoff, Friedrich, "Die Bedeutung der Sklaven für den Übergang von der Antike ins abendländische Mittelalter”, Historische Zeitschrift 192 (1961), 265-272.

VOGT, Joseph, Sklaverei und Humanität im klassischen Griechentum (Akademie der Wissenschaften und der Literatur Mainz. Abhandlungen der Geistes- und Sozialwissenschaftlichen Klasse 1953, 4), Wiesbaden 1953.

VOGT, Joseph, Sklaverei und Humanität. Studien zur antiken Sklaverei und ihrer Erforschung (Historia Einzelschriften 8), Wiesbaden 1972 [1e éd. 1965].

Wallace, Robert, A Dissertation on the Numbers of Mankind in Antient and Modern Times, Edimbourg 1753 [réimpr. Londres 1992].

Wallon, Henri Alexandre, Histoire de l'esclavage dans l'antiquité, 3 vols., Paris 1879 (réimpr. Aalen 1974) [1e éd. 1847].

WASSER, Hartmut, "«Wir halten den Wolf an den Ohren..." Thomas Jefferson und das Institut der Sklaverei”, Amerikastudien 41 (1996), 33-48.

Weber, Marianne, Max Weber. Ein Lebensbild, Tübingen 1926.

Weber, Max , Wirtschaftsgeschichte, éd. par Siegmund Hellmann/Melchior Palyi, Munich 1923 $(=\mathrm{Wg})$.

[WeBER, Max, Histoire économique. Esquisse d'une histoire universelle de l'économie et de la société. Traduit par Christian Bouchindhomme, préface de Philippe Raynaud, Paris 1991].

WebER, Max, Gesammelte Aufsätze zur Wissenschaftslehre, éd. par Johannes Winckelmann, Tübingen ${ }^{4} 1973$ (= GAWL).

[Weber, Max, Essais sur la théorie de la science. Traduit par Julien Freund, Paris 1965 (traduction partielle)] [réimpr. collection Agora Pocket, Paris 1992].

Weber, Max, Wirtschaft und Gesellschaft, éd. par Johannes Winckelmann, Tübingen $1980^{5}$ $(=\mathrm{WuG})$.

[Weber, Max, Économie et société. Traduit de l'allemand par Julien Freund, Pierre Kamnitzer, Pierre Bertrand, Eric de Dampierre, Jean Maillard et Jacques Chavy sous la direction de Jacques Chavy et d'Eric de Dampierre. Tome premier, Paris 1971 (traduction partielle)], [Réimpr. en 2 vols. en 1995 dans la collection Agora Pocket].

[Weber, Max, Sociologie du droit. Préface de Philippe Raynaud. Introduction et traduction par Jacques Grosclaude, Paris 1986.]

Weber, Max, Die Lage der Landarbeiter im ostelbischen Deutschland 1892, éd. par Martin RIESEBRODT, Tübingen 1984 (= MWG I/3).

Weber, Max, Zur Politik im Weltkrieg. Schriften und Reden 1914-1918, éd. par Wolfgang J. MOMMSEN, Tübingen 1984 (= MWG I/15).

[Partiellement dans : 
Weber, Max, Euvres politiques 1895-1919. Traduit de l'allemand par Elisabeth Kauffmann, Jean-Philippe Mathieu et Marie-Ange Roy. Présentation d'Elisabeth Kauffmann. Introduction de Catherine Colliot-Thélène, Paris 2004].

Weber, Max, Die römische Agrargeschichte in ihrer Bedeutung für das Staats- und Privatrecht 1891, éd. par Jürgen Deininger, Tübingen 1986 (= MWG I/2).

Weber, Max, Gesammelte Aufsätze zur Religionssoziologie, 3 vols [1920/21]. réimpr. Tübingen 1988 (= GARS).

[Sont cités ici les volumes suivants :

WEBER, Max, L'Éthique protestante et l'esprit du capitalisme, suivi d'autres essais. Édité, traduit et présenté par Jean-Pierre GrosseIN avec la collaboration de Fernand CAMBON, Paris 2003].

[WeBER, Max, Hindouisme et Bouddhisme. traduit par Isabelle Kalinowski avec la coll. de Roland Lardinois. Paris, 2003].

[Weber, Max, Le Judaïsme antique. Traduit par Freddy Raphael, Paris 1970].

Weber, Max, Gesammelte Aufsätze zur Soziologie und Sozialpolitik [1924], éd. par Marianne WEBER, réimpr. Tübingen 1988 (= GASS).

[Les passages cités sur la question de la race sont traduits dans : WINTER, Elke, Max Weber et les relations ethniques. Du refus du biologisme racial à l'État multinational. Suivi de : Le débat sur «race et société» au premier Congrès de la Société allemande de sociologie (1910), introduit par E. Winter, traduit par V. Wilkening et E. Winter, Les Presses de l'Université Laval 2004].

Weber, Max, Gesammelte Aufsätze zur Sozial- und Wirtschaftsgeschichte [1924], éd. par Marianne Weber, réimpr. Tübingen 1988 (= GASWG).

[Les textes sur l'Antiquité, contenus dans ce volume, se trouvent en traduction française dans :

Weber, Max (1999), Économie et société dans l'Antiquité. Précédé de : Les causes sociales du déclin de la civilisation antique. Introduction de Hinnerk Bruhns, Paris, Éditions La Découverte].

Weber, Max, Gesammelte Politische Schriften, éd. par Johannes WinCKELMAnN, réimpr. Tübingen 1988 (= GPS).

[Partiellement dans :

Weber, Max (2004), Euvres politiques, cf. supra.

WebER, Max, Landarbeiterfrage, Nationalstaat und Volkswirtschaftspolitik. Schriften und Reden 1892-1899, éd. par Wolfgang J. MOMMSEN, Tübingen 1993 (= MWG I/4).

WEBER, Max, Wirtschaft und Gesellschaft. Die Wirtschaft und die gesellschaftlichen Ordnungen und Mächte. Nachlaß. Teilband 5 : Die Stadt, éd. par Wilfried NIPPEL, Tübingen 1999 (= MWG I/22-5).

[Weber, Max, La Ville. Traduit par Philippe Fritsch. Préface de Julien Freund, Paris 1982].

WeBER, Max, Wirtschaft und Gesellschaft. Die Wirtschaft und die gesellschaftlichen Ordnungen und Mächte. Nachlaß. Teilband 1 : Gemeinschaften, éd. par Wolfgang J. Mommsen, Tübingen 2001 (= MWG I/22-1).

WeILER, Ingomar, Die Beendigung des Sklavenstatus im Altertum. Ein Beitrag zur vergleichenden Sozialgeschichte (Forschungen zur antiken Sklaverei 36), Stuttgart 2003.

WestermanN, William L., "Sklaverei”, in RE Supplement 6 (1935), 894-1063.

Wg, cf. WEBER, Max. Wirtschaftsgeschichte. 
Whittaker, Charles R./Garnsey, "Peter, Rural Life in the Later Roman Empire", in Averil Cameron/Peter Garnsey (éd.), The Cambridge Ancient History XIII : The Late Empire, A. D. 337-425, Cambridge 1998, 277-311.

WIELING, Hans (Bearbeiter), Corpus der römischen Rechtsquellen zur antiken Sklaverei, Teil I : Die Begründung des Sklavenstatus nach ius gentium und ius civile (Forschungen zur antiken Sklaverei, Beiheft 3), Stuttgart 1999.

WIRZ, Albert, Sklaverei und kapitalistisches Weltsystem, Francfort 1984.

Wish, Harvey, "Aristotle, Plato and the Mason-Dixon-Line", Journal of the History of Ideas 10 (1949), 254-266.

Woodman, Harold D., "The Profitability of Slavery. A historical perennial”, The Journal of Southern History 29 (1963), 303-325.

WuG, cf. Weber, Max. 